\title{
Interfaces in organic electronics
}

Mats Fahlman, Simone Fabiano, Viktor Gueskine, Daniel T Simon, Magnus Berggren and Xavier Crispin

The self-archived postprint version of this journal article is available at Linköping University Institutional Repository (DiVA):

http:// urn.kb.se/ resolve?urn=urn:nbn:se:liu:diva-160114

N.B.: When citing this work, cite the original publication.

The original publication is available at www.springerlink.com:

Fahlman, M., Fabiano, S., Gueskine, V., Simon, D. T, Berggren, M., Crispin, X., (2019), Interfaces in organic electronics, Nature Reviews Materials.

https:// doi.org/ 10.1038/s41578-019-0127-y

Original publication available at:

https:/ / doi.org/ 10.1038/ s41578-019-0127-y

N.B. The figures included in the original article are not included in the parallel published version

Copyright: Nature Research (part of Springer Nature) (Review J ournals) http:// www.nature.com/ 


\title{
Interfaces in organic electronics
}

Mats Fahlman $^{1}$, Simone Fabiano ${ }^{1}$, Viktor Gueskine ${ }^{1}$, Daniel Simon ${ }^{1}$, Magnus Berggren ${ }^{1,2}$ and Xavier Crispin ${ }^{1,2 *}$

${ }^{1}$ Laboratory for Organic Electronics, ITN, Linköping University, Norrköping, Sweden.

${ }^{2}$ Wallenberg Wood Science Center, ITN, Linköping University, Norrköping, Sweden.

*e-mail: xavier.crispin@liu.se

\section{Toc Blurb}

Organic semiconductors form clean interfaces with diverse materials, including metals, other organic semiconductors, electrolytes, dielectrics and biological organisms. In this Review, we discuss the properties of these interfaces and their central role in the function of organic electronic devices.

\begin{abstract}
Undoped conjugated organic molecules and polymers possess properties of semiconductors, including the electronic structure and charge transport, which can be readily tuned by chemical design. Moreover, organic semiconductors (OSs) can be n-doped or p-doped to become organic conductors (so called synthetic metals) and can exhibit mixed electronic and ionic conductivity. Compared with inorganic semiconductors and metals, organic (semi)conductors possess a unique feature: no insulating oxide forms on their surface when exposed to air. Thus, OSs form clean interfaces with many materials, including metals and other OSs. OS-metal and OS-OS interfaces have been intensely investigated over the past 30 years, from which a consistent theoretical description has emerged. Since the 2000s, increased attention has been paid to interfaces in organic electronics that involve dielectrics, electrolytes, ferroelectrics and even biological organisms. All these interfaces are central to the function of organic electronic devices, with the physico-chemical properties of the interfaces governing the interfacial transport of light, excitons, electrons and ions, as well as the transduction of electrons into the molecular language of cells.
\end{abstract}

\section{[H1] Introduction}

Organic semiconductors (OSs) can be used as the active material in diverse (opto)electronic devices and circuits. Compared with silicon-based electronics, organic electronics have many unique features, such as large optical absorption and emission, solution processability, mechanical flexibility and mixed ionic and electronic conduction. OSs comprise a broad family of semiconductors based on conjugated molecules or polymers (Fig. 1). The $\pi$ electrons of OSs form the valence and conduction bands. Upon reduction or oxidation, the $\pi$ system accommodates negative or positive charges, while counterions of opposite charge neutralize the overall material. Heavy doping leads to a dramatic change in the electronic structure, such that the bandgap vanishes and a Fermi level that lies between the occupied and unoccupied levels can be defined as in Fermi glasses ${ }^{1}$, metals ${ }^{2}$ or semimetals ${ }^{3}$. Undoped and doped OSs are therefore very different materials, but they share a unique feature: unlike inorganic semiconductors, no insulating oxide forms on their surface when exposed to air. Thus, OSs 
create clean interfaces with numerous materials, ranging from metals to biological organisms. The description of these interfaces is the focus of this Review.

We begin by describing the geometric and electronic structure of OSs, before providing an overview of organic electronics and the varied interfaces within. The remainder of the Review is divided into five parts, each related to a specific interface. In the two first sections, we discuss OS-metal and OS-OS interfaces. Over the past 30 years, these interfaces have been investigated in depth with many experimental solid-state characterization tools and, increasingly, advanced computational methods, such that fundamental theories have emerged. However, the interfaces between OSs and dielectrics, electrolytes and biological organisms are far less understood owing to their complexity and the experimental difficulties in characterizing them. Hence, these latter sections are primarily discussed in terms of state-of-the-art examples.

\section{[H1] Structure of organic semiconductors}

OSs are $\pi$-conjugated organic molecules or polymers with alternating single and double bonds. The $\sigma$ electrons form the (rather) planar skeleton, while the delocalized $\pi$ electrons create a halo of electronic density above and below the molecular plane (Fig. 1a,b). The frontier molecular orbitals - that is, the lowest unoccupied molecular orbital (LUMO) and highest occupied molecular orbital (HOMO) - have $\pi$ character (Fig. 1d). The $\pi$-electronic density of two adjacent molecules can interact weakly (Fig. 1e), and these $\pi-\pi$ interactions greatly affect the optical properties (for example, the size of excitons and the optical gap) and transport properties (such as mobility and the transport gap) of OSs. In an OS crystal, the interactions between LUMOs (HOMOs) of adjacent molecules can lead to the formation of a narrow conduction (valence) band in which the electrons (holes) are transported. The width of the bands is directly coupled to the strength of the $\pi-\pi$ interactions. These bands have a central role in determining the optical properties, oxidation and reduction potentials, chemical reactivity and electronic transport of OSs. In very narrow bands $(<0.2 \mathrm{eV})$, charge transport occurs through temperatureactivated hopping of localized charge carriers (with mobilities of up to $0.01-0.1 \mathrm{~cm}^{2} \mathrm{~V}^{-1} \mathrm{~s}^{-1}$ ). For wider bands, the transport of charge carriers is delocalized over many molecules in the $\pi$ $\pi$ stacks and resembles that of disordered inorganic semiconductors (with mobilities of up to $10-50 \mathrm{~cm}^{2} \mathrm{~V}^{-1} \mathrm{~s}^{-1}$ ). In some disordered semiconducting polymers, there is local order; a few molecules organize into a percolation path (Fig. 1f), which ensures a moderately high mobility $\left(\sim 1 \mathrm{~cm}^{2} \mathrm{~V}^{-1} \mathrm{~s}^{-1}\right)$ within the solid.

Compared with non-conjugated molecules, conjugated molecules are typically characterized by a low ionization potential (IP) and a high electron affinity (EA). The IP and EA (Fig. 1d) are often defined as IP $=E_{\text {vac }}-E_{\mathrm{HOMO}}$ and $\mathrm{EA}=E_{\mathrm{vac}}-E_{\mathrm{LUMO}}$, respectively (where $E_{\mathrm{vac}}$ is the vacuum level and $E_{\text {HOMO }}$ and $E_{\text {LUMO }}$ are the energies of the HOMO and LUMO, respectively). However, the oxidation and reduction energies of the solid phase of OSs are additionally modified by both intramolecular and intermolecular screening, which depend strongly on the (local) film order. Molecules with a low IP (high EA) are called electron donors (acceptors) and are easily oxidized (reduced) — that is, positively (negatively) doped — and thus form p-(n)type OSs. Typically, the excess doping charges carried on a conjugated molecule are localized to a specific region, which is characterized by a change in the bond length alternation compared with the neutral molecule. These charge defects are called (positive or negative) polarons (for a charge of \pm 1 ) or bipolarons (for a charge of \pm 2 ; Fig. 1c), and the associated electronic states are located in the bandgap of the material. As the electrical conductivity depends on the product 
of mobility and charge concentration, an OS that is heavily oxidized (with up to $30 \%$ of the monomers carrying a charge) can reach conductivities of up to several thousand $\mathrm{S} \mathrm{cm}^{-1}$ (to date, heavily reduced OSs have reached conductivities of only a few $\mathrm{S} \mathrm{cm}^{-1}$ ). Such OSs with high conductivities are referred to as organic conductors (or synthetic metals). To maintain electroneutrality, anions or cations are intercalated within the bulk of the p-doped and n-doped OSs, respectively. The intercalation of ions in OSs is favoured by the weak intermolecular interactions (van der Waals, electrostatics and $\pi-\pi$ interactions). In a doped OS film swelled with solvent, both electronic and ionic charge carriers are mobile (Fig. 1g), which is crucial for many applications.

\section{[H1] Interfaces in organic electronics}

Organic light-emitting diodes (OLEDs) are the first products based on OSs to reach the market ${ }^{4}$. Within an OLED, electrons and holes are injected by metal (or metal oxide) electrodes, forming excitons (hole-electron pairs) in the undoped OS that recombine to emit light (Fig. 2a). There has been a substantial effort to understand the energetics of the interfaces between metals (or metal oxides) and OSs. To lower the operating voltage of OLEDs, holetransport layers with a low IP (or electron-transport layers with a high EA) made of either undoped $^{5}$ or doped ${ }^{6}$ OSs are added between the emitting OS and the electrode contact (Fig. 2a). The energetics at the OS-OS interface between organic charge-transport layers and the organic emitting layer is central to achieve efficient exciton recombination. OLEDs have reached very high total external efficiencies ( 20\%) through the design of interfacial energetics and by finding strategies, such as phosphorescence ${ }^{7}$ and delayed fluorescence ${ }^{8}$, to create light from the normally non-radiative triplet excitons formed by $75 \%$ of the injected charge carriers. OLED devices are typically fabricated through vacuum deposition of conjugated molecules onto clean metal surfaces or vice versa. A possible alternative is to use printing technology to deposit thin films with soluble emitting polymers ${ }^{9}$. OLEDs have been explored for flexible ${ }^{10}$ and even stretchable $^{11}$ colour displays and white-light sources ${ }^{12}$.

Organic photovoltaics (OPVs) are another class of devices based on OSs and are close to reaching the market ${ }^{13}$. The active layer is sandwiched between two electrodes and is composed of an interpenetrated network of two different OSs, one with electron-donor character (high IP) and the other with electron-acceptor character (low EA) (Fig. 2b). Photons that cross the transparent anode (for example, indium tin oxide) are absorbed by the active layer and produce excitons. Subsequent exciton dissociation produces an electron current that runs through the acceptor network to reach the cathode and a hole current that travels through the donor network to the anode. Like OLEDs, OPVs can be further optimized through the inclusion of charge-transporting layers to promote and filter charge collection at the two electrodes ${ }^{14}$. OPVs have motivated researchers to reconsider the role of the interface between two different OSs as efficient exciton separation occurs at the interface between nanodomains of electrondonor and electron-acceptor molecules. The energetics of the OS-metal and OS-OS interfaces is crucial in achieving high efficiency, and the number of interfaces increases in state-of-the-art tandem solar cells that optimize the absorption of the solar spectrum. OPVs have reached power conversion efficiencies of up to $17.3 \%$ for solution-processed tandem solar cells ${ }^{15}$. A life-cycle cost analysis of OPVs indicates that this technology is economically viable and sustainable for achievable ratios between lifetime and power conversion efficiency ${ }^{16}$. Solution-processable OSs have a shorter energy payback time compared with silicon-based photovoltaics owing to 
their less energy-intensive manufacturing processes, mostly enabled by printed electronics and low-temperature chemical synthesis ${ }^{14}$. Advances in computational power have enabled an unprecedented level of description of the interfaces (OS-metal ${ }^{17}$ and OS-OS ${ }^{18}$ ) in OPVs, enabling interface modelling and engineering to progress from conceptual to quantitative.

In addition to the fabrication of OPVs, printed electronics is emerging as a viable manufacturing method for diverse organic electronic devices. This manufacturing process is enabled by the solubility of OSs, which allows for the formulation of semiconducting inks for printing (opto)electronic devices on flexible substrates (such as paper, plastic and textiles) ${ }^{19}$. The printing machines generally operate in air, and thus the surface of any metal electrode is typically coated by either an oxide and/or a sub-monolayer of impurities that passivate the surface $^{20}$. Hence, OSs interact weakly with such electrodes, typically through physisorption rather than chemisorption ${ }^{21}$. The technology rush on printed electronics, motivated by a huge emerging market for the internet of things, necessitates the rapid development of OS-electrode interfaces to increase device performance.

One of the cornerstone components of organic electronics, printed ${ }^{22}$ or flexible, is the transistor. Organic field-effect transistors (OFETs) are needed to create logic circuits and also smart pixels in displays ${ }^{23}$. An OFET is a three-terminal device in which the current flowing between the source and drain through the OS channel is controlled by the electric potential drop through the dielectric layer that separates the channel and the gate contact (Fig. 2c). The nature of the interface between the dielectric and the OS has a key role in determining the mobility and electrical characteristics of the transistor ${ }^{24,25}$. The interface between the source or drain and the OS layer governs the contact resistance and determines whether the current passing through the OFET is limited by charge injection or charge transport in the OS channel. It is imperative to design the materials and device architecture of an OFET to reach high field-effect mobility, because this is related to both the clock frequency of a circuit and to the amount of current that can be delivered to a pixel in a display. The interface between the OS and the dielectric is the most important region in an OFET. Indeed, the accumulation of charges in the channel is localized within approximately one monolayer of the OS thin film from the interface with the dielectric. This localization explains why a channel made of a single self-assembled monolayer (SAM) is sufficient to obtain transistor function ${ }^{26}$. Hence, the properties of the interface (morphology, traps and dielectric permittivity $(k)$ ) can strongly affect the charge-carrier mobility ${ }^{27}$. Owing to the removal of traps at the OS-dielectric interface by chemical design, electron and hole mobilities of the same order of magnitude are now achievable in $\mathrm{OSs}^{28}$. Today, high electron and hole mobilities have been obtained in both organic single crystals ${ }^{29}$ (10-50 $\mathrm{cm}^{2} \mathrm{~V}^{-1} \mathrm{~s}^{-1}$ ) and solution-processed semiconducting polymers ${ }^{30}$ (up to $3 \mathrm{~cm}^{2} \mathrm{~V}^{-1} \mathrm{~s}^{-1}$ ). The small difference in charge-carrier mobility between single crystals of small organic molecules and semicrystalline polymers is explained by the dominant role of short-range order for the transport of charges ${ }^{31}$. The choice of the dielectric is not only based on the charge-carrier mobility in the OS, but also on the energy consumption of the device. In terms of energy consumption, several strategies have been proposed to reduce the operating voltage of OFETs, including the formation of a monolayer-thick dielectric ${ }^{32}$, the use of a high- $k$ dielectric $^{33}$ or even the formation of an electric double layer (EDL) upon polarization of an electrolyte ${ }^{34}$. Today, owing to optimization of the OS and the OS-dielectric interface, OFETs are competitive with amorphous silicon thin-film transistors. Functionalization of the dielectric can lead to other 
interesting features of an OFET, such as memory function originating from the bistability of the polarization in ferroelectric polymer dielectrics ${ }^{35}$.

The mixed conduction of electronic and ionic charge carriers leads to a peculiar interface when an OS is in contact with an electrolyte. An electrolyte is a highly polarizable medium that can result in the formation of an EDL in the vicinity of the OS (Fig. 2c). This EDL creates a large local electric field that can be used for charge storage ${ }^{36,37}$, charge transport ${ }^{34,38}$ or light emission ${ }^{39,40}$. The degree of interpenetration of the OS-electrolyte interface can vary between two extremes. At one extreme, a well-defined interface between the two layers at the monolayer level can be achieved with an undoped hydrophobic OS coated by a polyanionic electrolyte. In this case, no anions penetrate into the positively biased OS and a well-defined EDL is created only at the interface ${ }^{41}$. This OS-electrolyte interface quickly charges $(\sim 10-$ $100 \mu \mathrm{s})$ an EDL, reaching $\sim 10 \mu \mathrm{F} \mathrm{cm}^{-2}$, which is advantageous for creating low-voltage $(<1 \mathrm{~V})$ electrolyte-gated transistors ${ }^{42}$ (Fig. 2c). However, an interface between a hydrophilic electrolyte and an undoped hydrophobic semiconductor is challenging to fabricate because dewetting occurs. Other material combinations have been found to stabilize the interface, such as a hydrophobic ionic gel ${ }^{43}$, although ions from the gel can penetrate into the OS layer and slow the transistor ${ }^{34}$. At the other extreme, a diffuse interface forms between a blend of a positively charged conducting polymer balanced with a polyanion and a polyanionic electrolyte layer. In this case, anions from the electrolyte penetrate the OS, and thus the OS-electrolyte interface is an interpenetration of phases; this 3D bulk interface enables a large specific capacitance, which is advantageous for supercapacitors ${ }^{36}$. In a typical conducting-polymer supercapacitor (Fig. 2d), two metallic collectors inject electronic charges into the doped OS layers, while the electrolyte provides the ionic charges that penetrate into the OS layers to balance the electronic charge. Between these two extreme cases are blends of an undoped OS with an electrolyte that contains both mobile cations and anions. When such an ionic-electronic conductor is sandwiched between two electrodes, the electric polarization leads to the progressive formation of a negatively doped region in the material at the cathode and a positively doped region at the anode. The two doped regions grow and meet to form a neutral 'insulating' region, that is, a self-formed $\mathrm{p}-\mathrm{i}-\mathrm{n}$ device ${ }^{40}$. Electrons and holes are injected and light emission takes place from this neutral region. This phenomenon is exploited in organic electrochemical emitting cells ${ }^{39}$, ${ }^{40}$. An additional feature of organic mixed ionic-electronic conductors is that they can transduce an electrical signal into an ionic signal. Signal transduction is the key principle when interfacing electronics with living organisms that have organic ion pumps, as their languages are different: electrons on the one hand and ions ${ }^{44}$ or ionic molecules, such as neurotransmitters ${ }^{45}$, on the other hand. Hence, the interface between an OS and a biological organism appears to be a new interface with many possibilities for organic electronics.

Finally, the development of organic electronics is moving in new directions, such as organic thermoelectrics ${ }^{46,47}$, bioelectronics ${ }^{48,49}$, electrocatalysis ${ }^{50,51}$ and cellulose-based electronics ${ }^{52,53}$. As OSs have a small $\mathrm{CO}_{2}$ footprint, largely due to their low-temperature processability, there is an emerging push towards 'green electronics,' which considers, for example, eco-toxicology, biocompatibility and biodegradability. The properties of organic interfaces will thus be of great (and likely increasing) interest in the foreseeable future.

\section{[H1] Semiconductor-metal interfaces}


OS-metal interfaces are ubiquitous in organic electronics, typically serving either to inject or extract charges from an OS film. The alignment of the charge-transporting states in the OS with the Fermi level of the metal is thus of key importance to device functionality. However, the charge-injection and charge-extraction barriers cannot typically be estimated by comparing the work function of the clean metal surface with the bulk EA and IP values of the OS ${ }^{21,54}$ owing to several effects that arise when an OS is deposited onto a metal surface. Each of these effects produces an interface dipole potential step, $\Delta$ int, that shifts the vacuum level at the metal contact (Fig. 3a). We start by using the example of an atomically clean metal surface to discuss each of these effects in turn.

\section{[H2] Interface dipole potential steps.}

The work functions of metals are readily available in the literature, although these are typically for atomically clean surfaces of a particular crystal orientation. This is important to note as the metal work function is derived from the bulk chemical potential and the electrostatic potential across the metal surface, the latter resulting from an excess of electron density outside the metal surface (known as dangling bonds), which is compensated by an electron density deficiency inside the metal surface to achieve charge neutrality ${ }^{55}$. This electrostatic potential energy step can be $>1 \mathrm{eV}$ and depends on the surface crystal orientation. The electrostatic potential energy (and hence the work function) is also affected by the adsorption of heteroatoms and molecules on the metal surface even in absence of chemisorption, as has been demonstrated using noble gas atoms ${ }^{56}$. Surface adsorption compresses the electron density tail that extends out of the metal surface and decreases the surface electrostatic potential step. This decrease in the metal work function upon formation of an OS film or absorption of, for example, oxygen or hydrocarbons, is often referred to as the push-back effect and is always present at OS-metal interfaces ${ }^{57}$. The push-back dipole step, $\Delta_{\mathrm{pb}}$, always decreases the work function and is often significant. For gold, a $\Delta_{\mathrm{pb}}$ of $\sim 0.7 \mathrm{eV}$ was obtained regardless of the deposition order (that is, organic-on-gold or gold-on-organic), yielding an effective work function for gold of $\sim 4.5 \mathrm{eV}$, which is generally unsuitable for both electron and hole injection ${ }^{58}$.

The adsorption of OSs onto a clean metal surface leads to hybridization of the frontier molecular orbitals and partial charge transfer between those orbitals and the metal density of states (DOS) ${ }^{57,59}$. This chemisorption-induced through-bond charge transfer creates an interfacial dipole layer and induces an additional shift of the vacuum level, $\Delta_{\text {chem }}$, at the OS-metal interface. Electron transfer from the metal to the OS upshifts the vacuum level (increasing the effective work function), whereas electron transfer from the OS to the metal downshifts the vacuum level (decreasing the effective work function). The degree of orbital hybridization is dependent on the strength of chemisorption: for strong chemisorption, hybridization gives rise to completely new mixed metal-organic orbitals whereas for weak chemisorption, hybridization merely broadens and shifts the original molecular orbitals ${ }^{21}$. 59 . OSs typically adsorb weakly on clean coinage metal surfaces but adsorb strongly on clean transition metal surfaces.

Adsorbed molecules or polymers with intrinsic dipole moments can produce a dipole potential step, $\Delta_{\text {intdp }}$, if the adsorbed layer has a preferential order, which is the case for SAMs, for example. If the intrinsic molecular dipole moments are oriented towards the metal surface, 
the vacuum level is upshifted, whereas a downshift results from the opposite orientation of the molecular dipole moments ${ }^{21}$ 57. However, owing to depolarization effects between the individual dipole moments in the surface layer, the effective dipole moment that each molecule contributes to the overall dipole potential step is reduced ${ }^{60}$. Under certain conditions, dipole moments can be induced in both the organic overlayer and the metal, together yielding a socalled double-dipole potential step, $\Delta_{\mathrm{ddp}}$ (refs ${ }^{61,62}$ ). If the organic layer contains charged species (for example, anions and cations), the metal surface is polarized by these charged species, forming image charges. The charged species in the organic layer are attracted to their respective image charges and move towards the metal surface. If one of the charged species is more restricted than the other in its ability to approach the metal surface, there will be a notable difference in the final equilibrium positions at the substrate surface. A difference in the equilibrium positions induces a dipole moment between the charged species in the adsorbed organic layer, which in turn induces a dipole moment in the metal substrate and creates a $\Delta_{\text {ddp }}$ in the vacuum level at the OS-metal interface (Fig. 3b). If the anions are the more mobile of the two charged species, they will (on average) come closer to the metal surface than the cations, downshifting the vacuum level (Fig. 3b, right). Correspondingly, if the cations are more mobile than the anions, the cations form the more intimate contacts to the metal surface and the vacuum level is upshifted (Fig. 3b, left). As well as ions, a $\Delta$ ddp can also be induced by an organic layer containing tertiary aliphatic amines, which have a notable dipole moment owing to the lone pair of electrons on the nitrogen atom, and positive and negative polarons in a molecularly doped OS film ${ }^{61,62}$. Nevertheless, for all cases, the range of the attractive Coulomb force from the image charges is limited, and thus the double-dipole-step effect is significant only for the layer directly adsorbed on the metal surface ${ }^{61,62}$. The induced $\Delta_{\mathrm{ddp}}$ can be quite large; for example, poly[(9,9-bis(3'-(N,N-dimethylamino)propyl)-2,7-fluorene)-alt-2,7-(9,9-dioctyl-fluorene)] (PFN) typically yields a downshift of $0.3-0.8 \mathrm{eV}$ for metal contacts ${ }^{61}$, making PFN an efficient interlayer material for producing low-work-function electrodes in, for example, organic solar cells.

So far, we have described the vacuum level shifts that can be introduced when a clean metal surface is covered by an OS monolayer; however, these shifts can also be induced by a native oxide or hydrocarbon layer. Indeed, as organic electronic devices are typically manufactured under ambient conditions, in a glovebox or under high vacuum $\left(>10^{-7} \mathrm{mbar}\right)$, the metal surfaces will generally be covered by a native oxide or hydrocarbon layer prior to the deposition of the OS, rendering the metal surfaces chemically inert. Hence, OS films fabricated under these conditions, physisorb onto a passivated metal surface that has an effective work function determined by the vacuum level shifts ${ }^{21}$ discussed above. For OS films fabricated under ultrahigh vacuum conditions $\left(\sim 10^{-9} \mathrm{mbar}\right)$, the first layer chemisorbs, but the subsequent layers physisorb on the monolayer-passivated metal surface. OS-metal interfaces formed by physisorption can still feature large vacuum level shifts, as discussed below.

Following deposition of an OS film on a metal surface, the chemical potential equilibrates throughout the multilayer structure. The equilibration results from a flow of charge across the OS-metal interface, leading to a potential energy gradient that shifts the vacuum level. This equilibration also occurs for physisorbed OS films provided that the passivating layer on the metal surface is not completely insulating or too thick, thus preventing tunnelling. The charge that equilibrates the chemical potential can come from doping or defect-induced DOSs that 
exist in the organic layer prior to contacting the metal and determine the chemical potential in the OS film. Alternatively, the charges can be generated upon metal contacting through spontaneous oxidation or reduction of the molecules or polymers in the OS. In the former case, the potential energy gradient follows the depletion-region model from classical semiconductor physics (with occasional exceptions at low doping levels). Thus, there is an extended region (a depletion region) in the organic film away from the interface in which the vacuum level (and hence also the conduction and valence bands) bends either upwards (for hole transfer to the metal) or downwards (for electron transfer to the metal). This bending occurs for all interfaces except when the initial chemical potentials of the (modified) metal and OS are equal. However, the DOS induced by doping an OS film does not directly correspond to a free charge density that can be used to equilibrate the chemical potential. The electrostatic interaction between the dopant charge and the corresponding charge on the organic molecule or polymer is sufficiently strong to ensure that a considerable portion of the doped charge density is bound and localized in the vicinity of the dopant molecule or ion (particularly for low doping levels) ${ }^{63}$. The depletion region in doped OS films thus typically extends further than would otherwise be assumed for a given doping concentration, permittivity and initial chemical potential difference.

For the high-quality purified OS materials used in organic electronic devices, however, the more relevant scenario is the absence of notable doping or defect-induced free charge DOS. In this case, the chemical potential is equilibrated by spontaneous oxidation or reduction of molecules to form polaronic states at the interface and subsequent integer charge transfer (ICT) across the interface via tunnelling; thus, pre-existing free charges do not have a major role ${ }^{21,64-}$ ${ }^{67}$. As oxidation and reduction energies (that is, the formation energies of positive and negative polarons, respectively) are highly dependent on local intermolecular and intramolecular order ${ }^{68}$, ${ }^{69}$, the occupied and unoccupied DOS at the interface will typically differ from that of the bulk, as interface formation often affects film order. Furthermore, owing to the generally low relative permittivity of OS films, there is a strong electrostatic interaction between the interfacial polarons created upon equilibration of the chemical potential and the image charges in the metal substrate, and thus there is a significant Coulombic contribution to the energies of the ICT states (that is, interfacial polarons electrostatically coupled to the substrate image charges). Hence, the distributions of oxidation and reduction energies are shifted into the energy gap in the OS layers near the metal interface (Fig. 3a). As a consequence, the Fermi level becomes pinned above (below) the bulk IP (EA), which often limits the depletion region to 1-2 layers ${ }^{70}$. The resulting energy level alignment for this type of interface can be divided into three general cases: a downshift, $\Delta_{\mathrm{ICT}}$, of the vacuum level as the chemical potential in the OS film becomes pinned to a value, $E_{\mathrm{ICT}+}$, located at the edge of the $\mathrm{ICT}^{+} \mathrm{DOS}$ and hence above the bulk valenceband edge when the passivated metal work function $\Phi_{\text {met }}>E_{\text {ICT }+}$; vacuum level alignment $\left(\Delta_{\text {ICT }}=0\right)$ for $E_{\text {ICT- }}<\Phi_{\text {met }}<E_{\text {ICT }+}$; and an upshift, $\Delta_{\text {ICT }}$, of the vacuum level as the chemical potential in the OS film becomes pinned to a value, $E_{\text {ICT-}}$, located at the edge of the ICT ${ }^{-}$DOS and hence below the bulk conduction-band edge when $E_{\text {ICT- }}<\Phi_{\text {met }}\left(\right.$ ref. $\left.^{21}\right)$. As the pinning energies, $E_{\text {ICT }}$ and $E_{\text {ICT- }}$, and the ICT DOS that they are derived from are highly dependent on the Coulomb energy contribution, they can be tuned by manipulating the electrostatic interaction between the induced charges in the organic interface layer and the image charges on the metal surface (Fig. 3c). By inserting SAMs with thicknesses of up to $3 \mathrm{~nm}$, the $E_{\text {ICT- }}$ of an $\mathrm{OS}$, and hence the electron-injection barrier, can be decreased by $0.3 \mathrm{eV}$ compared with the 
corresponding intimate OS-metal contact ${ }^{71}$. Polyelectrolyte cathode interlayers also decrease $E_{\text {ICT- }}$ in addition to inducing a $\Delta_{\text {ddp }}$ of the cathode work function. This electrostatic decoupling has more recently been demonstrated in devices in which wide bandgap and high IP anode interlayers, tris(4-carbazoyl-9-ylphenyl)amine and (4,4'-bis( $N$-carbazolyl)-1,1'-biphenyl), with thicknesses of $\sim 3-5 \mathrm{~nm}$ were used to increase $E_{\text {ICT }}$ for a series of OSs, bringing $E_{\text {ICT }}$ closer to the bulk IP values and thereby improving the hole-injection efficiency by more than an order of magnitude ${ }^{72}$.

\section{[H2] Computational modelling.}

Computational methods are increasingly used to calculate and model the properties of OS-metal interfaces and are highly useful for the design of OSs and devices. Density functional theory (DFT) is the main approach for determining the ground-state electronic structure of OS molecules as well as the hybridized states and partial charge transfer resulting from metal-OS chemisorption. The hybridized states created by chemisorption depend on the bonding site(s) of the metal surface and the orientation of the molecules. These parameters can be obtained from experiment or through modelling, although the latter can be demanding in terms of computational effort. For weak chemisorption, the DFT-calculated DOS is typically broadened with a Lorentzian function to simulate the hybridization of the frontier orbitals rather than performing a complete calculation of the metal-organic interaction for all molecules in the film. This broadened DOS together with the metal work function is then used to determine the degree of charge transfer and the resulting vacuum-level shift at the interface ${ }^{59,67}$.

The energy level alignment of films at physisorbed OS-metal interfaces are controlled by their ionized (or excited) states, which are in turn dependent on the intermolecular and intramolecular order that typically varies both laterally and vertically in an OS film. Multiscale modelling approaches can be used to obtain the film morphology and the corresponding DOS starting from calculated properties (such as molecular geometries and partial charges) of the individual molecules ${ }^{73}$ but are computationally very demanding. Thus, film disorder and related effects are often modelled using a Gaussian broadening of the DOS. Moreover, many computational approaches make use of the fact that the strong electrostatic interaction between the charged molecules and metal image charges typically confines the charge transfer to the first OS layer so that an EDL is formed. Hence, the interface can be approximated as a plane capacitor from which the interface potential step is readily calculated ${ }^{64-66}$. Potential gradients that extend several layers into the organic film can be treated using a similar approach, in which the layer thickness and DOS are introduced individually for each layer. Using this approach, it is possible to vary both the position and width of the DOS depending on the distance between the organic layer and the substrate, enabling effects such as image-charge interaction and intralayer order variations to be simulated ${ }^{67}$. The occupied (unoccupied) DOS is obtained from calculated IP (EA) energies that are broadened using a Gaussian function of a chosen width for physisorbed interfaces or a Lorentizan function when simulating weakly chemisorbed layers. The layer-by-layer potential gradient in the OS film is then obtained using Poisson's equation ${ }^{67}$.

The recent advances in computational methods now enable accurate calculations of the energy level alignment for OS-metal systems in which the intermolecular order is well defined and known, owing to the interdependence between intermolecular (and intramolecular) order and the oxidation and reduction energies. For true predictive power and the in silico 
design of OS films and OS-metal interfaces, a key remaining challenge is the continued development of cost-effective multiscale modelling methods for the determination of the properties of film formation and the resulting interfacial and bulk DOS for a given OS and metal surface, as well as for large (polymeric) materials.

\section{[H1] Semiconductor-semiconductor interfaces}

As the surfaces of OS films are largely non-reactive, chemisorption with bond formation and partial charge transfer is usually absent from OS-OS interfaces (with some exceptions, discussed below). Weak (van der Waals) interactions typically dominate at OS heterojunctions, and the chemical potential is thus equilibrated by the $\Delta_{\text {ICT }}$ induced by the oxidation of molecules on one side of the interface and the reduction of molecules on the other. Just as for the OS-metal interfaces, the strong electrostatic interactions between the, here, oppositely charged molecules across the heterojunction limits the $\Delta_{\text {ICT }}$ potential energy gradient to 1-2 layers on either side of the interface but can extend further owing to, for example, depthdependent variations in intermolecular order and a high concentration of (defect or dopinginduced) free charges in the layer(s). Intermolecular order is of particular importance for OSOS heterojunctions featuring small molecules, as these can form highly ordered films. For example, a layer (or part of a layer) in which the molecules lie flat with respect to the substrate can have very different oxidation and reduction energies compared with a layer in which the same molecules are oriented perpendicular to the substrate ${ }^{69,74}$, considerably affecting the values of $E_{\text {ICT }}$ and $E_{\text {ICT- }}$ and hence the energy level alignment ${ }^{74}$. For the same reasons, local defects in the order of a crystalline film can also influence the energy level alignment ${ }^{75}$.

ICT is the main, but not the only, factor that determines the energy level alignment at OS-OS heterojunctions. Highly ordered (crystalline) layers of OSs with molecular quadrupole moments will not only have different oxidation and reduction energies (and hence different $E_{\text {ICT }}$ and $\left.E_{\text {ICT- }}\right)$ depending on the crystal orientation, but if interfaced with a layer of an OS that lacks molecular quadrupole moments (for example, a pentacene- $\mathrm{C}_{60}$ interface), the resulting discontinuity of the quadrupole field at the heterojunction can produce an additional interface dipole step $\left(\Delta_{\text {intdp }}\right)$ that shifts the vacuum level ${ }^{76}$. Moreover, for small-molecule OS-OS heterojunctions, orbital hybridization can have a notable role in determining the energy level alignment. For example, a molecular dipole moment is created by the partial charge transfer between the donor and acceptor, introducing an interface dipole potential step $\left(\Delta_{\text {chem }}\right)$. Weak hybridization is typically modelled computationally using a Lorentzian broadening of the frontier density of states, similar to the modelling of weak hybridization at OS-metal interfaces ${ }^{59,67}$. Additionally, donor (such as quaterthiophene) and acceptor (such as tetracyanoquinodimethane) molecules can form charge-transfer complexes in which strong electronic coupling leads to orbital hybridization and partial (rather than integer) charge transfer $^{77,}{ }^{78}$. For strong hybridization, a ground-state charge-transfer complex is formed, resulting in a supramolecule with a new set of frontier orbitals ${ }^{77-79}$. Hybridization requires specific spatial overlap between the orbitals of the participating molecules and is thus typically restricted to well-ordered small-molecule films and heterojunctions. Other forms of strongly interacting OS-OS interfaces also exist. For example, hydrogen bonding between molecules at an interface can affect the energy level alignment both directly through modification of the DOS and indirectly by modifying the intermolecular order. For the particular case of interfaces between fullerenes and acenes, bond-forming chemisorption can occur (through Diels-Alder cycloaddition reactions), as recently demonstrated for pentacene- $\mathrm{C}_{60}$ (ref. ${ }^{80}$ ).

Organic electronic devices often contain several thin-film layers to provide various functionalities, and the chemical potential must then be equilibrated across the whole multilayer stack, including each interface. We illustrate this using trilayer structures (metal- 
OS-OS) and assume physisorbed interfaces ${ }^{81-83}$. First, we consider a metal-donor-acceptor trilayer, in which the metal-donor interface is in vacuum level alignment (Fig. 4a). To equilibrate the chemical potential, charge is transferred across the donor-acceptor interface, building up an interface potential step. The maximum potential step at this interface (given by the energy difference between $E_{\text {ICT- }}$ of the acceptor and $E_{\text {ICT+ }}$ of the donor, $\left.E_{\text {ICT-,A }}-E_{\text {ICT+,D }}\right)$ is not sufficiently large to cause equilibration, so additional charge is transferred from the metal to the acceptor layer, contributing to a linear potential gradient over the donor layer; this has been experimentally demonstrated for aluminium-tetrathiafulvane-tetracyanoquinodimethane multilayer stacks ${ }^{81}$. By contrast, for a metal-donor interface that is pinned to $E_{\text {ICT+,D, }}$ the equilibration of the chemical potential is facilitated by charge transfer at the donor-acceptor interface, and hence there is not a significant potential gradient over the donor layer (Fig. 4b). We now reverse the situation and consider metal-acceptor-donor trilayers and again assume physisorbed interfaces. When the metal-acceptor interface is vacuum level aligned, the addition of the donor layer induces charge transfer across the acceptor-donor interface, downshifting the vacuum level (Fig. 4c). Again, the maximum potential step at this interface ( $E_{\text {ICT-,A }}-$ $E_{\text {ICT }+ \text { D }}$ ) is not sufficiently large to cause equilibration, so additional charge is transferred from the donor layer to the metal, contributing to a linear potential gradient over the acceptor layer. Finally, for a metal-acceptor layer for which the Fermi level is pinned to $E_{\text {ICT-,A, the }}$ introduction of the donor layer equilibrates the chemical potential across the stack by charge transfer across the acceptor-donor interface (Fig. 4d). These cases illustrate that the energy level alignment at OS-OS (and OS-metal) interfaces depend on the position of the chemical potential and thus is not solely determined by the relationship between the donor IP and acceptor EA at the OS-OS heterojunction.

The nature of the interface and energy level alignment at OS-OS heterojunctions have notable effects on the performance of organic electronic devices. For light-emitting devices, organic heterojunctions can be used to create exciplexes with tuned effective bandgaps and minimized singlet-triplet energy splitting (realized through the physical separation of donor and acceptor molecules across the interface), suitable for either direct thermally activated delayed fluorescence or as triplet-harvesters for embedded fluorescent emitters ${ }^{84}$. For OPV devices, the OS-OS heterojunction is of great importance, as the open circuit voltage, $V_{\text {oc }}$, correlates with the effective energy gap of the donor-acceptor interface ${ }^{85-87}$. Thus, the absence or presence of an interface dipole potential step and the mechanisms through which the dipole step was created have key roles in determining device performance.

A monolayer of molecules with intrinsic dipole moments can be inserted between donor and acceptor layers that would otherwise have vacuum level alignment. When the orientation of the molecules in the dipole layer is such that the collective dipole field upshifts the vacuum level ${ }^{88}$ (Fig. 5a), the LUMO of the acceptor is shifted upwards relative to the donor HOMO, increasing the effective donor-acceptor bandgap and by extension the $V_{\text {oc }}$. Furthermore, it has been shown theoretically and experimentally that a dipole layer at a donoracceptor interface with the negative charge facing the acceptor and positive charge facing the donor increases the dissociation of photogenerated charge-transfer excitons ${ }^{89}$, 90 , which is of benefit to photovoltaic devices. By contrast, for a donor-acceptor interface for which chemical equilibration and upshift of the vacuum level occurs by an $\Delta_{\text {ICT }}$ that arises from oxidation of donor molecules and the corresponding reduction of acceptor molecules, there is a density of interfacial ICT states (these are acceptor negative (n) polarons electrostatically bound to donor positive (p) polarons) even under dark conditions ${ }^{91}$ (Fig. 5b). Again, the vacuum level shift increases the effective bandgap compared with vacuum level alignment, and the dipole layer is directed such that photogenerated charge-transfer exciton dissociation is enhanced. The $\Delta_{\text {ICT }}$ case differs from the $\Delta_{\text {intdp }}$ case, however, in that the ICT DOS can act as charge recombination sites for photogenerated free p-polarons and n-polarons and decrease the $V_{\text {oc }}$ through trap- 
assisted recombination ${ }^{91}$. This effect can be large in bulk heterojunction structures, as has been experimentally demonstrated for a series of poly(3-hexylthiophene) (P3HT)-fullerene

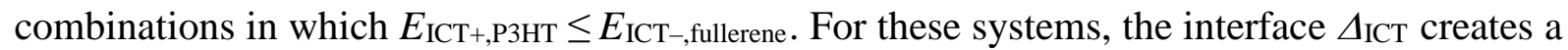
nearly constant effective bandgap across the series. However, there is a large variation in the $V_{\text {oc }}$ owing to an increase in the density of ICT states, and hence the trap-assisted recombination, as $\Delta_{\text {ICT }}$ increases ${ }^{91}$. The effective bandgap can also be increased when a dipole step is introduced by a discontinuity of a quadrupole field at a donor-acceptor interface, as has been calculated for interfaces between face-on lying pentacene and $\mathrm{C}_{60}\left(\right.$ ref. $\left.^{76}\right)$. Within pentacene$\mathrm{C}_{60}$, a dipole potential step is introduced at the interface, with the $\mathrm{C}_{60}$ molecules being negatively polarized and the pentacene molecules positively polarized. The potential step thus causes an upshift of the vacuum level and an increase in the effective bandgap, which should facilitate dissociation of photogenerated charge-transfer excitons. Finally, although the focus here has been on physisorbed interfaces, effects from chemisorption at OS-OS interfaces, albeit rare, do occur. For example, the defects introduced by bond formation at fullerene-acene interfaces substantially modify the photovoltaic performance ${ }^{92}$.

In recent years, there have been great advances in the measurement and understanding of the time-dependent formation and evolution of (photo)excited states at OSOS heterojunctions as well as their effect on the performance of $\mathrm{OPVs}^{93-97}$, seemingly overturning long-held beliefs, such as the need for a substantial driving force to achieve efficient free-charge generation ${ }^{93,97,98}$. In comparison, understanding of the precise relationship between effects such as exciton-to-free-charge generation and energy loss and the ground-state electronic structure and energy level alignment at OS-OS heterojunctions is lacking, and a challenge is to obtain accurate measurements of the EA of OS materials. Inverse photoemission spectroscopy (IPES) provides a direct measurement of the EAs of both OS monolayers and films, but the radiation-sensitive nature of the OS materials and limitations in IPES energy resolution have greatly hindered research efforts. Recent advances in instrumentation and methodology for high-resolution and low-radiation-dose IPES show great promise for solving these problems ${ }^{99}$, which would greatly aid both model development and device design.

\section{[H1] Semiconductor-dielectric interfaces}

The interface between an OS and a dielectric has a fundamental role in the operation of OFETs $^{100}$. The central processes in these devices, such as charge accumulation and transport, occur near the interface between the OS and the gate dielectric layer. Thus, understanding how the physico-chemical properties of the insulator affect the structural and energetic disorder of OSs at the interface is vital for maximizing the figures of merit of the devices ${ }^{101}$.

\section{[H2] Surface energy and morphology.}

The surface chemistry and texture of the dielectric can affect the molecular order and thin-film morphology of the OS, causing severe degradation of the transport properties ${ }^{102,103}$. As the OS layers are typically deposited under non-equilibrium conditions, the resulting film morphology is the outcome of non-equilibrium processes ${ }^{104}$, such as nucleation, growth, wetting and crystallization $^{105-107}$, that take place at the interface between the OS and the dielectric layer. The surface energy and morphology of the dielectric layer influence the diffusion, aggregation and crystallization of the organic (macro-)molecules on top of the dielectric surface, thus affecting the structural quality of the OS layer ${ }^{108-110}$. Adjusting the surface tension of the insulator layer by grafting, for example, a SAM onto the dielectric surface ${ }^{111}$, is an effective route to control the molecular orientation ${ }^{112,}{ }^{113}$, grain size and grain boundary density ${ }^{114,} 115$ and 
polymorphism ${ }^{116-118}$ of both solution-processed and vacuum-deposited OSs, resulting nearly always in improved OFET performance (Fig. 6a).

In addition to the chemical interactions, the texture and dynamics of the underlying substrate also greatly affect the assembly of OSs. Smooth, defect-free dielectric surfaces typically lead to large grain sizes and reduced grain boundaries, which thus result in a low trap density and increased mobility ${ }^{119-121}$. By contrast, mechanically rubbed substrates featuring grooved patterns in the direction of transport can guide the alignment of subsequently grown polymer thin films, yielding thin-film anisotropy and enhanced charge transport ${ }^{122-124}$. The viscoelasticity of the dielectric surface also has a large impact on the assembly of vapourdeposited and solution-processed OSs, influencing the OS film growth and microstructure across different length scales and increasing charge-carrier mobilities along both the $\pi-\pi$ stacking and polymer backbone directions ${ }^{125,126}$. When the dielectric layer is deposited on the OS film by wet processes, as in a top-gated OFET architecture, other effects need to be taken into account. For example, chemical and physical deterioration of the active materials by the dielectric solvent and the conditions for the process may result in chemical doping or degradation of the underlying semiconductor film ${ }^{127,}{ }^{128}$. Comprehensive reviews on gate dielectric materials and their impact on the semiconductor morphology in OFETs can be found elsewhere $^{129-131}$.

\section{[H2] Dipolar electrostatic disorder.}

The polarity of the insulating layer not only affects the structural quality of the OS, in which the charge carriers are transported, but also influences its electronic structure and the DOS. Varying the dielectric permittivity of the gate insulator can result in mobilities that differ by more than one order of magnitude. Although the use of highly polarizable gate dielectrics, such as those based on high- $k$ oxides ${ }^{132}$, allows for low-voltage operation ${ }^{133}$, it also promotes strong charge-dipole coupling, which induces the localization of charge carriers and the formation of interfacial Fröhlich polarons ${ }^{27}$. As a result of charge localization, the charge-carrier mobility decreases upon increasing the dielectric permittivity of the gate insulator and has a temperature dependence that evolves from metallic-like to insulating-like as the dielectric permittivity increases. When polymeric insulators with a permittivity in the range 2-4 are instead used as the dielectric materials, the charge-dipole coupling is not strong enough for interfacial polarons to form. In this case, the charge-transport mechanisms are dominated by so-called dipolar (energetic) disorder, which originates from the randomly oriented dipole moments in the gate dielectric $^{134}$. These dipoles generate potential fluctuations in the channel material that broaden the DOS of the OS. As the OS-dielectric interface becomes more polar, the broadening of the DOS becomes more severe, increasing the localization of carriers at the interface ${ }^{135}$. The degree of energetic disorder induced by the charge-dipole interaction varies strongly with distance from the interface ${ }^{136}$, and charge modulation spectroscopy measurements reveal that dipolar disorder in high- $k$ insulators enhances the localization of polarons at the interface more than those in the bulk ${ }^{137}$ (Fig. 6b). By contrast, in low- $k$ polymeric dielectrics, the polarons at interfaces have a similar degree of localization to those in the bulk ${ }^{137}$. For disordered (amorphous) OSs, the broadening results in a decreased DOS at the Fermi energy, a reduced hopping probability and thus a decrease in charge-carrier mobility ${ }^{138}$. Similar observations have been reported for highly ordered OSs that display band-like transport, suggesting that dipolar 
disorder affects the DOS of OSs even when charge transport is not mediated by hopping ${ }^{139}$. Note that the molecular structure of the OS has a large effect on suppressing the coupling between the charge carriers in the channel and the electrical polarization in the gate dielectric. For example, fluorocarbon chains linked to a $\pi$-conjugated core screen the electrons that accumulate in the first layer from the gate insulator and from the nearby $\pi$-conjugated layers within the crystal, which both influence the dielectric environment through their polarizability, resulting in band-like transport ${ }^{140}$.

\section{[H2] Charge trapping.}

The OS-dielectric interface can also influence the operational stability of the OS by providing sites for charge trapping ${ }^{141}$. The commonly used silicon oxide dielectric, for example, is both a homogeneous passive substrate and an active surface at which electroactive sites, such as hydroxyl groups, can act as traps and hinder electron transport ${ }^{142}$. Nonpolar SAMs or polymeric coatings can be used to modify the dielectric surface and minimize the density of surface trap sites, thereby increasing mobility and even enabling ambipolar operation ${ }^{143}$. Similar observations have also been reported for gate polymeric insulators that contain hydroxyl groups, such as polyvinylphenol, indicating that the presence of acidic functional groups in the insulating material can chemically trap mobile electrons at the interface ${ }^{144}$ (Fig. 6c). Decreasing organic contamination at the OS-insulator interface by means of surface treatment, such as with oxygen plasma ${ }^{145}$, decreases the number of interfacial trap sites, thus yielding superior device performance. Water molecules physisorbed to the dielectric surface can also trigger charge trapping, thereby inducing bias-stress instability ${ }^{146,147}$ (Fig. 6d). Treatment of the dielectric surface with hydrophobic SAMs can be an effective way to passivate these electrochemically active defects and to hinder the physisorption of water or other contaminants, thus reducing bias-stress instability ${ }^{148-150}$. However, it is necessary to be cautious with the choice of SAM and especially the polarity of their end groups, which influences the ability of the SAM to trap charges in the channel region. Polar SAMs carrying electron-withdrawing (such as fluorine) or electron-donating (such as $\mathrm{NH}_{2}$ ) end groups can permanently trap charges, as demonstrated by measurements of surface potential with scanning Kelvin probe microscopy ${ }^{151}$. Exposure of polymer-based gate dielectrics to humidity results in increased ion migration in the gate dielectric, causing the threshold voltage to shift. Polar polymer gate dielectrics, such as poly(vinylphenol) ${ }^{152}$, poly(vinyl alcohol) ${ }^{153}$ and poly(styrenesulfonic acid) ${ }^{154}$, are particularly prone to this effect owing to their hygroscopic character and high concentration of ions, whereas nonpolar low- $k$ polymer dielectrics, such as Cytop (a fluoropolymer) ${ }^{155}$, do not usually exhibit this behaviour.

\section{[H2] Surface dipole potential.}

In addition to charge trapping, interface dipoles in the gate dielectric and/or SAMs on the dielectric surface can also modify the charge-carrier density in the semiconductor channel, inducing appreciable shifts in the threshold voltage and increasing carrier mobility ${ }^{156-158}$. For instance, fluorinated SAMs grafted onto a $\mathrm{SiO}_{2}$ dielectric surface typically enhance the holecarrier density in the channel of pentacene-based OFETs ${ }^{156}$, thus shifting the threshold voltage to more positive values and increasing the field-effect mobility (Fig. 6e). The opposite trend is observed for n-type fullerene-based transistors. These properties can be understood in terms of 
electrostatic charge-dipole interactions and weak charge transfer between the OS and the SAM molecules. Note that in top-gated OFETs, chemical functionalization of the bottom substrate also influences charge transport, leading to variation in the threshold voltage and surface carrier density $^{159}$. Moreover, hole transport and injection in a predominantly n-type semiconducting polymer can be dramatically increased by using a fluorinated high- $k$ insulating polymer as the gate dielectric material ${ }^{160}$. The increase in $\mathrm{p}$-channel characteristics originates from the $\mathrm{C}-\mathrm{F}$ dipoles at the dielectric surface and not from bulk effects. Therefore, the OS-dielectric interface can effectively modulate channel polarity in OFETs, thus leading to efficient complementary electronic circuits ${ }^{161}$.

\section{[H2] Functional interfaces.}

As discussed so far, small changes at the OS-insulator interface causes dramatic changes in the electrical properties of OFETs. Thus, functionalization of this interface can provide an effective way to improve device performance and, most importantly, to introduce new functionalities ${ }^{162}$. For example, reversible changes in the dipole moment of photoactive SAMs, triggered by light of different wavelengths, produce two distinct built-in electric fields at the OS-insulator interface that can modulate the channel conductance and consequently the threshold voltage, thus leading to functional memory devices ${ }^{163}$. However, charges that accumulate at this interface are essentially volatile, that is, they are only present as long as an external gate voltage is applied. Reversible trapping-de-trapping of charges in the gate dielectric or at its interface with the OS can promote non-volatile memory functionalities. For example, electrons transferred from pentacene to a polymeric gate dielectric can yield excellent non-volatile memory characteristics, such as large memory windows, reversible and fast switching and long retention times ${ }^{164}$. The magnitude of the memory window is strongly affected by the hydrophilicity and dielectric polarity of the polymeric gate insulator ${ }^{165}$. Charge-trapping can be optimized by introducing specific trap sites, such as metal nanoparticles or charge-accepting (and typically $\pi$-conjugated) molecules, into the dielectic ${ }^{166}$. Organic ferroelectric gate insulators with permanent and/or switchable electrical dipoles offer another approach to introduce non-volatile charge transport in OSs ${ }^{167}$. Because of the inductive effect of fluorine atoms, a large dipole moment exists in fluorinated polymers such as poly(vinylidene fluoride) and its derivatives. These dipole moments collectively align under the influence of an electric field through rotation of the monomer groups within the crystalline domains, giving rise to a ferroelectric response. The surface dipole density of the ferroelectric layer subsequently induces a large and permanent accumulation of charges in the semiconductor channel. Polarizing the ferroelectric dipoles in the opposite direction depletes the channel of charges (for unipolar semiconductors) ${ }^{168}$ or accumulates charges of opposite sign (for ambipolar semiconductors) ${ }^{169}$, ${ }^{170}$. The result is a sharp change of the OFET channel conductance at the coercive field of the ferroelectric gate (Fig. 6f). The ferroelectric polarization can also be transferred to an electrolyte $^{171}$, which in turn induces electrostatic charge accumulation and/or electrochemical doping in an organic (semi-)conductor placed in contact with it, enabling passive matrix addressing of electrochromic displays ${ }^{172}$ and advanced memory functionalities in artificial synapses $^{173}$.

\section{[H2] From 2D to 3D interfaces.}


Blending OSs with polymeric insulators has emerged as a general strategy to improve their optoelectronic performance ${ }^{174}$, processability $^{175}$ and mechanical ${ }^{176}$ and environmental ${ }^{177}$ stability. In addition to tuning the viscoelastic ${ }^{175}$ and optical ${ }^{178}$ properties of the semiconducting ink, the insulating medium confines the semiconducting phase to nanometre-scale dimensions, enabling effective control of long-range (crystallinity) and short-range (aggregation) order ${ }^{179}$ to achieve efficient charge transport. The dilution of OSs in wide-bandgap insulators also decreases the number of transport sites and traps, yielding single-crystal-like performance in solution-coated OFETs ${ }^{180}$ and balanced electron and hole transport as well as reduced nonradiative trap-assisted recombination in OLEDs ${ }^{181}$. The confinement also affects the mechanical stretchability of otherwise brittle polymeric semiconductors. Owing to a finite-size effect and interface effect, confining a polymer into an insulating medium enhances polymer chain dynamics in the amorphous regions ${ }^{182}$, and the restricted growth of large crystallites ${ }^{183}$ enables high stretchability in semiconducting materials ${ }^{184}$. Interpenetrating networks comprising semicrystalline conjugated polymers and insulating polymers with a high glasstransition temperature can also confine conformational changes of the semiconducting polymer chains at elevated temperatures, resulting in semiconducting active layers that display temperature-insensitive charge-transport behaviour up to $220^{\circ} \mathrm{C}$ in OFETs ${ }^{185}$. Another important aspect of blending OSs with an insulator is that this can lead to self-encapsulation of the resulting active layer, increasing air stability compared with neat semiconductors ${ }^{186,187}$. To exploit the beneficial effect of blending an OS with an insulating polymer, various aspects need to be considered, such as the molecular weight of the materials, the degree of crystallinity of the insulating polymer, the crystallization sequence and crystallization kinetics. This strategy is not limited to polymer:polymer blends and can also be used for insulating polymer:smallmolecule semiconductor ${ }^{188,189}$ blends or copolymers comprising semiconducting and insulating moieties $^{190,191 .}$

\section{[H1] Semiconductor-electrolyte}

Owing to the initial focus on the changes resulting from carrier injection into the polymer chains of OSs, the presence of counterions (that is, ions of opposite charge to the OS molecules) was ignored in early theoretical models. However, electroneutrality cannot be bypassed, and the discovery of the electroactivity of OS polymers ${ }^{192,193}$ placed the OS-electrolyte interface in the spotlight. Indeed, doping-dedoping is accompanied by the movement of counterions in the vicinity and through the OS-electrolyte interface. The electrochemical doping-dedoping of OSs involves bulk as well as surface processes, and their interplay affects the kinetics and reversibility of changes, both crucial for the operation of electronic devices.

Depending on the method of preparation, the initial state of an OS can be either undoped (that is, neutral, pristine and semiconducting) or doped (that is, charged, containing counterions and conducting). Small-molecule OSs (such as rubrene) and many conducting polymers (for example, P3HT) are prepared in the neutral state and are then chemically or electrochemically doped $^{194}$. However, conducting polymers synthesized by oxidative polymerization (chemical or electrochemical) are intrinsically p-doped during the synthesis. The mode of operation of an OS in a device comprising an electrolyte depends on the initial state of doping and on the possibility of ion exchange between the electrolyte and the OS bulk. Injection of ions through 
the interface depends on the accessibility of the OS bulk to species from the solution, which in turn depends on the size of the ions and on the morphology of the OS. If the ions from solution do not cross the phase boundary with the polymer, their action is limited to the EDL at the interface. By contrast, if the polymer bulk is accessible to the ions (Fig. 7), changes in the charge state of the OS upon doping or dedoping are compensated by absorption or expulsion of counterions (Fig. 7a,b), or by expulsion or absorption of co-ions (that is, ions of the same charge as the OS; Fig. 7c,d); in both cases, the ions are accompanied by solvent molecules. The latter process, that is, co-ion movement, is particularly relevant when bulky counterions incorporated into a doped conducting polymer remain immobilized upon charge-discharge. This is the case for one of the most popular and commercially successful conducting polymers, poly(3,4ethylenedioxythiophene):polystyrene sulfonate (PEDOT:PSS), which is synthesized by oxidative polymerization and is thus prepared in a p-doped form with the polyelectrolyte PSS providing charge-compensating immobile anions. Upon electrochemical activity of PEDOT:PSS, charge neutrality is achieved exclusively by the movement of cations across the OS-electrolyte interface.

As immobile ions are usually present in the charged OS, the so-called Donnan phenomenon manifests at the OS-electrolyte interface ${ }^{195}$ : the immobile ions prevent the penetration of mobile co-ions from the electrolyte, and a potential difference is established at the interface (known as the Donnan potential). Although the Donnan phenomenon in the OS context was recognized in the 1990s (for example, ref. ${ }^{196}$ ), a complete understanding of the effect has only been achieved in the past few years ${ }^{197}$. In practice, the Donnan phenomenon manifests as a dependence of the electrochemical response of an OS on the ionic strength of the electrolyte as well as the concentration of active ions and can cause a potential drop of up to $100 \mathrm{mV}$ across the OS-electrolyte interface at low electrolyte concentrations.

Experimentally, the properties of OS-electrolyte interfaces and changes during dopingdedoping can, in principle, be determined using electrochemical impedance spectroscopy (EIS). However, meaningful physical interpretation of the data relies on fitting to the behaviour of model electrical circuits, the choice of which is not unique ${ }^{198}$. The study of OSs using EIS is further complicated by the roughness, porosity and semiconducting character of the surfaces ${ }^{199}$. Thus, it is encouraging that in the case of PEDOT:PSS dedoping, a physical picture of a cation front advancing into the polymer and the hole front consequently receding offers a satisfactory explanation of the EIS data ${ }^{200}$. Another approach to understand the phenomenon taking place upon electrochemical activity of an OS is optical tracking of the kinetics of the moving front of ions inside of an OS. This approach is possible because of the difference in optical properties of the doped and dedoped forms ${ }^{201}$. It was noted recently that EIS in conjunction with photoelectrochemistry can provide an experimental platform for efficient studies of OSelectrolyte interfaces; however, such data are scarce ${ }^{202}$.

Electrochemically induced doping-dedoping has been extensively exploited to create new technological opportunities. For example, it is possible to go beyond traditional electronic devices by exploiting ionic-electronic mixed conduction, such as in electrochromic displays ${ }^{203}$, light electrochemical cells $s^{39}, 40$ and electrokinetic devices ${ }^{204}$. However, the role of the OS- 
electrolyte interface has not been the main focus in these areas. By contrast, the OS-electrolyte interface has been studied more intensively for electroactuators, wettability switches, charge storage and electrochemical transistors, as discussed below.

\section{[H2] Electroactuation.}

Ion and solvent absorption or expulsion cause changes in the OS volume (Fig. 7), an effect that has led to the development the field of actuators and artificial muscles $\underline{205} \underline{206}$. Purely electrochemical data cannot discriminate counterion and co-ion transport through the interface. However, powerful techniques, such as the electrochemical crystal microbalance (EQCM), enable material flow through OS-electrolyte interfaces to be followed. EQCM is sensitive to mass changes of the electrode with nanogram precision, which, together with electrochemistry, can reveal the charge of compensating ions and their overall quantity, corresponding to the passing charge, as well as the quantity of accompanying solvent. EQCM was applied early in the study of conducting polymer electrochemistry ${ }^{207}$ and has become a standard tool. In situ atomic force microscopy enables the swelling of an organic layer on an electrode to be monitored, revealing local inhomogeneities that occur during the process ${ }^{208}$. More recently, similar capabilities were demonstrated by electrochemical strain microscopy, and it was shown that amorphous regions of an OS polymer swell owing to the penetration of counterions, whereas the stiffer, more crystalline domains do not expand ${ }^{209}$.

\section{[H2] Surface switch.}

The surface wettability of the conducting polymer PEDOT:PSS can be tuned by dipping the polymer surface in an ionic liquid. The change in wettability (from an initial wetting contact angle of $48^{\circ}$ to a contact angle of $100^{\circ}$ ) is due to the exchange of surface protons with the large hydrophobic organic cations of the ionic liquid ${ }^{210}$. An interesting yet understudied effect in the context of ion and solvent penetration into the OS bulk is the change in hydrophilicity during electrochemical doping at the OS-electrolyte interface. Indeed, an undoped organic material is often hydrophobic, but its hydrophilicity can increase when it becomes charged. Thus, a poly(3alkylthiophene) layer in an electrolyte can be reversibly electrochemically switched between a dedoped hydrophobic state (with a contact angle of $105.9^{\circ}$ ) and a doped hydrophilic state (with a contact angle of $\left.76.7^{\circ}\right)^{211}$, and this phenomenon has been reviewed elsewhere ${ }^{212}$. Therefore, in an aqueous solvent, the accessibility of the bulk to solvated ions can be, in principle, increased through doping. The electrochemical switching of wettability at the OS-electrolyte interface can be implemented in microfluidic devices to control the wettability of the walls of the microfluidic channels and thus the flow of liquids ${ }^{213}$. A metallic mesh functionalized with an OS layer can act as a valve that controls whether a liquid can pass depending on the voltage applied to the mesh ${ }^{214}$. Besides wettability, some reports point to the possibility of triggering the dissolution of OS layers upon electrochemical oxidation or reduction, although the mechanisms are not fully understood ${ }^{215} 216$.

\section{[H2] Charge storage.}

The coexistence of the interfacial and bulk charge-discharge phenomena of OSs is the apparent origin of their complex electrochemical characteristics, which evade simple classification into battery-like (Faradaic) or capacitive behaviour (Fig. 8). A cyclic voltammogram (CV) is the 
trace of current, $I$, recorded as a function of voltage, $V$, linearly changing with time, $t$. The current response owing to capacitive charging at the interface in cyclic voltammetry is featureless, potential independent $(I=C \mathrm{~d} V / \mathrm{d} t$, where $C$ is the capacitance) and box-like (Fig. 8a). A redox reaction manifests in a $\mathrm{CV}$ as a pair of peaks, an anodic peak (oxidation) and a cathodic peak (reduction) (Fig. 8b), the positions of which are determined by the reversible potential of the reaction and other factors (such as reversibility and the mass transport regime). A typical CV of an OS resembles a superposition of both types of response, with Faradaic-like peaks, which are often rather wide, superimposed on capacitive-like boxes (Fig. 8c).

The relative contribution of the Faradaic and capacitive response of an OS polymer can be related to its porosity. Through nanopores, small ions insert between the polymer chains, so that the OS film with ions constitutes a homogeneous thermodynamic phase $\mathrm{e}^{217}$; therefore, the OSelectrolyte interface is situated at the outer OS surface. In the framework of this single-phase model, the equilibrium potential drop is predicted to be distributed between both the metal-OS and OS-electrolyte interfaces. In the case of low doping, the potential drop is also distributed through the OS film (as the doping level decreases from the OS-electrolyte towards the metalOS interface). Therefore, only a fraction of the applied polarization is translated into a potential drop at the OS-electrolyte interface, where many of the important phenomena occur. For macropores, which are typically larger than $10 \mathrm{~nm}$, small ions form a macroscopic OSelectrolyte interface; in this case, the OS film can be considered as a two-phase system with a very large specific surface. The formation of an EDL at the 3D bulk OS-electrolyte interface enables a large specific capacitance, which is advantageous for supercapacitors ${ }^{36}$.

For the complex case of PEDOT:PSS, the traditional view relating charge storage in an OS polymer to a sum of Faradaic and capacitive processes was challenged in a recent theoretical study $^{218}$. The electrochemical response of this polymer in a wide potential range was accounted for by pure capacitance, originating from the EDLs formed along the interfaces between nanoscale PEDOT-rich and PSS-rich grains that constitute the two phases of bulk PEDOT:PSS. The entire system, when including solvent inside the polymer film, therefore comprises three phases. However, nanoscale systems can be said to exhibit Faradaic pseudocapacitance, because a Faradaic (redox) reaction of the redox centres located near the surface, that is at a distance $\mathrm{l} \ll(2 D t)^{1 / 2}$ (where $D$ is the diffusion coefficient for charge-compensating ions), is electrochemically indistinguishable from purely capacitive, double-layer charging ${ }^{219}$. Furthermore, this behaviour, along with the data accumulated from various other solid-state battery and capacitor electrodes, led to the development of the 'supercapattery' concept, marrying the traditional supercapacitor and battery ${ }^{220}$.

\section{[H2] Electrolyte-gated transistors.}

Electrolyte-gated transistors are not only technologically relevant, but are also the devices that reveal the most about the properties and influence of the OS-electrolyte interface. Indeed, an electrochemical transistor with an OS as active layer of the transistor channel was invented just a few years after the discovery of these materials ${ }^{221}$. 
In an OFET (Fig. 2c), the electronic current passing through the OS channel between the source and the drain electrodes typically depends on the gate voltage, which is established between the channel and the gate electrode. The gate/electrolyte/OS channel has a capacitor-like structure, and the gate bias triggers the doping-dedoping of the OS channel and thus modulates the electrical conductivity. The gate electrode is not in direct contact with the channel, but exerts its influence through an electrolyte. The role of the electrolyte is two-fold: it is necessary for driving electrochemical reactions that involve the OS channel, and it forms EDLs at the interfaces with the channel and the gate with an extraordinarily high capacitance of up to $500 \mu \mathrm{F} \mathrm{cm}^{-2}$ (ref. $^{41}$ ).

Depending on whether ions from the electrolyte can penetrate into the OS film, two limiting types of OFETs can be discerned: organic electrochemical transistors (OECTs) and electrolytegated organic field-effect transistors (EGOFETs). Within an OECT, the interface is ionpermeable, and the operation relies on the exchange of ions between the electrolyte and the bulk of the OS and thus on its electrochemical doping-dedoping, which requires charge compensation $^{222}$. If the OS channel is initially in a doped state, the transistor is in its ON state without applying a gate voltage and will operate in depletion mode, in which it is gradually switched OFF (dedoped) as the gate voltage increases. The entire thickness of the OS channel is accessible for doping-dedoping reactions, as has been shown for PEDOT:PSS through the linear scaling of the transconductance and capacitance with volume ${ }^{223}$. If the pristine state of the OS channel is undoped, the transistor is in its OFF state and will function in the accumulation mode (that is, the gate voltage will accumulate charge carriers in the channel) ${ }^{224}$. OECTs can operate as n-type and p-type transistors with small ions penetrating a pristine neutral semiconductor $^{225}$.

When the interface is ion-blocking, either because the channel material is dense and nonporous, or because the electrolyte contains only bulky or chemically incompatible ions, the device is called a EGOFET, and it is more similar to an ion-sensitive FET or even a traditional FET than to an OECT. The use of an ion-blocking interface limits the operation of the transistor to the accumulation mode. The penetration of ions from the electrolyte into the OS phase can be prevented by using a solid-state polyanionic proton-conducting electrolyte in a p-type EGOFET $^{41}$ and a solid-state polycationic layer in a n-type EGOFET ${ }^{226}$.

For both types of transistor, the changes in channel conductivity are triggered by the polarization of the electrolyte. Three distinct electrolyte polarization mechanisms can be discerned at different timescales: dipolar relaxation at high frequencies, ionic relaxation (migration) at intermediate frequencies and EDL formation at the electrolyte interface at low frequencies. The ideal electrolytes for achieving a quick and intense response are a solid electrolyte with high ionic mobility and fast polarization even in a dry environment or a polymeric ionic liquid ${ }^{227}$. Upon polarization of the electrolyte, the response of the OS, that is, electric-field tuning of the charge density, should be considerable. The highest charge accumulation in an EGOFET, on the order of $10^{13} \mathrm{~cm}^{-2}$ without breakdown, was reported not for an OS polymer, but for single crystals of rubrene at low temperatures that had a large surface capacity at the electrolyte (an ionic liquid in this case) interface. The large surface capacity 
enables control of the surface conductivity over many orders of magnitude ${ }^{228}$. Among the electrolytes that provide very high capacitances, ion-gel gate dielectrics (which are triblock copolymers swollen with ionic liquids) stand out. Ion gels have achieved capacitances of up to $100 \mu \mathrm{F} \mathrm{cm}^{-2}$ due to a high concentration of mobile ions that assures the formation of an EDL only nanometres in thickness ${ }^{229}$.

In general, an EGOFET is characterized by fast response times owing to rapid restructuring of the EDL, while an OECT enables high currents and transconductance (defined as $g_{\mathrm{m}}=\partial I_{\mathrm{D}} / \partial V_{\mathrm{G}}$, where $I_{\mathrm{D}}$ is the drain current and $V_{\mathrm{G}}$ is the gate voltage), as the channel material is dopeddedoped in the OS bulk and not only at the OS-electrolyte interface ${ }^{223}$. The mode of operation (OECT or EGOFET) is not determined solely by the properties of the materials but also depends on the operation parameters. For example, timescales (that is, for how long the gate voltage is applied) can also be crucial for determining the mechanism of operation as they determine whether counterions from the electrolyte have sufficient time to penetrate into the semiconductor ${ }^{229}$; in some cases, the penetrability can be controlled by the chemistry of the interface ${ }^{230}$. The operation mode of an organic transistor can also be determined by controlling the penetrability of small ions into the OS film; this can be achieved by engineering the hydrophilicity or hydrophobicity of the OS through the OS molecule or polymer side chains. For example, a thiophene-based polymer with hydrophobic side chains is impenetrable to chloride in aqueous solution, whereas its close analogue with a similar morphology but with more hydrophilic chains manifests reversible electrochromism and a large modulation in capacitance owing to the increased accessibility of the bulk for ion exchange.

The interface between the gate electrode and the electrolyte should not be forgotten. Indeed, the gate electrode also functions as the counter electrode, leading to the division of the applied gate-voltage drop between both the OS-electrolyte and gate-electrolyte interfaces. When the charge compensated at the OS-electrolyte interface is not high, a Faradaic reaction at the counter (gate) electrode is not necessary and can be replaced by a capacitive polarization. However, if the capacity at the gate electrode is low, the major part of the gate potential drops at its interface with the electrolyte rather than at the OS-electrolyte interface. This situation can be remedied by ensuring that the Faradaic reaction at the gate electrode is highly reversible and thus non-polarizable, such as is the case for silver cations in an aqueous solution that contains chloride $^{231,232}$. Another way to influence the performance of the OS-electrolyte interface is to tune the capacity of the gate (counter) electrode by modifying its morphology. With a nanoporous high-capacity gate, the entire electroactive OS film in the channel can be oxidized without the need of a second half-reaction at the counter electrode. By contrast, a flat counter electrode limits the electrochemical reaction of the OS to the interface with the electrolyte, thus creating a confined EDL. Thus, by gate engineering, the behaviour of the transistor is switched between the OECT mode (for a high-capacity gate) and the EGOFET mode (when limited by a low gate capacity) ${ }^{233}$.

\section{[H1] Semiconductor-biological organisms}


Ions (including metal ions, $\mathrm{Cl}^{-}$and $\mathrm{H}^{+}$) have an important role in the communication between cells and their environment. Biomolecules and transmembrane proteins serve as charge carriers and enable selective signal recognition and amplification. Furthermore, cells release adhesion-promoting proteins, such as fibronectin, that contain signalling sequences (such as arginine-glycine-aspartate (RGD)). These sequences support or suppress integration and connectivity by providing attachment sites to other cells and the extracellular matrix. The surface and bulk of organic electronic materials not only transport electronic charge carriers, but can also express, transport and even release ions and charged biomolecules. This ability is central to the function of interfaces between OSs and biological organisms (that is, OS-bio interfaces). Moreover, through covalent and ionic interactions, proteins and other bioactive species can be immobilized along the bare surface of an OS. Together, these features make OSbio interfaces a unique and particularly important asset in bridging the signalling gap between biology and technology. Importantly, although the adhesion between OSs and biological systems can be controlled by various chemical and physical interactions, there is generally an electrolytic phase between the OS and biological system. Hence, OS-bio interfaces typically include both OS-electrolyte and electrolyte-biological interfaces.

\section{[H2] Cell and tissue adhesion along organic semiconductor surfaces.}

Chemically or electrochemically polymerized conducting polymers, such as PEDOT and PPy, have been explored for direct interfacing with cells, such as neurons, and whole neural tissues, such as the brain. Although pristine films of PPy ${ }^{234-236}$ and PEDOT ${ }^{237-239}$ have good biocompatibility, there are several strategies to further improve surface biocompatibility or to make the electronic interface preferential for specific cell types. CDPGYIGSR is a nine-aminoacid fragment of laminin (an extracellular matrix protein) that promotes the attachment of neurons. This signal sequence has been co-deposited with both $\mathrm{PEDOT}^{240}$ and $\mathrm{PPy}^{236}$ onto electrode surfaces, resulting in the favourable growth of neuroblastoma cells on sites functionalized with CDPGYIGSR. In comparison, sites coated with PPy doped with $\mathrm{CH}_{3} \mathrm{COO}^{-}$ showed no specific preference for cell growth. The electropolymerization of ethylenedioxythiophenes with different functional groups (such as alcohol, carboxylic acid and activated $N$-hydroxysuccinimidyl esters) in aqueous microemulsions (Fig. 9a) has been explored to fabricate functionalized PEDOT films for bioconjugation ${ }^{241}$. This approach facilitates the orthogonal growth of thin $(<100 \mathrm{~nm}$ ) and very smooth (roughness $<5 \mathrm{~nm}$ ) films. Moreover, by varying the composition of monomers, the surface properties of the films can be tuned for bioconjugation, and the surfaces exhibit low intrinsic cytotoxicity and little inflammatory response upon implantation. More recent strategies to increase the biocompatibility of organic electronic surfaces include the development of composites containing, for instance, chitosan ${ }^{234}$ or polydopamine ${ }^{242}$, the fine-tuning of the elasticity ${ }^{243}(0.3-$ $2.2 \mathrm{kPa}$ ), inclusion of RGD peptides ${ }^{244}$ and the incorporation of lysine side chains ${ }^{245}$.

\section{[H2] Organic bioelectronic electrode surfaces.}

A biocompatible organic electronic surface could be included in an array of electrodes and devices to record and regulate biological processes. The electrode surface is the simplest device structure, and several early experiments were conducted to explore organic electrodes for intimate contact with biological systems and biomolecules. In 1996, researchers seeded 
aortic endothelial cells on bare PPy electrode surfaces that were switched between the oxidized and neutral states ${ }^{235}$. Regardless of the oxidation state of the surface, a high degree of protein adsorption was observed; however, along the oxidized PPy surface, cells adhered and spread normally, whereas along the neutral PPy surface, both cell extension and DNA synthesis were suppressed. It was hypothesized that upon redox switching, the release of dopant ions from the PPy surface generates an osmotic shock that affects cell-growth processes. In 2009, similar experiments were reported in which PEDOT-tosylate surfaces were shown to regulate the adhesion and growth of epithelial cells on electrodes ${ }^{239}$. The origin of electroactive control over cell growth was found to stem from redox-induced changes in the conformation of fibronectin (Fig. 9b), which adsorbs along the surface. Along the reduced surface, fibronectin adopts a favourable conformation that exposes the RGD sequence, promoting integrin coupling and resulting in cell binding and the formation of polarized monolayers. Conversely, along the oxidized surface, the fibronectin conformation buries the RGD motif; thus, only a few cells adhere and no proliferation is observed ${ }^{239}$. As organic electrodes are being explored to record and initiate signalling in neuronal cells, there is a great need to improve the organic electroniccell interface with respect to biocompatibility and impedance matching.

\section{[H2] Volumetric organic bioelectronic surfaces.}

The planar 2D surface of organic electronics is very different from the natural geometry of (neuronal) cells. Several strategies have been applied to promote better interfacing by developing various 3D structures or scaffolds that provide a non-stratified, non-planar interface with biological organisms. PPy has been electrochemically grown into and through hydrogel layers deposited on top of microelectrodes to form volumetric electrodes (that is, 3D structured porous electrodes) with improved impedance characteristics for neuronal recordings ${ }^{246}$. Conducting polymer electrodes that exhibit mixed ion-electron charge transport, such as PPy and PEDOT electrodes, are typically nanostructured or microstructured and have a large volumetric capacitance ${ }^{218,223,247}$ (Fig. 9c). The large volumetric capacitance results from the formation of ion-electron double layers throughout the entire volume of the electrode and enables the impedance of the electrode to be matched to that of cell surfaces, for example, of depolarized neurons. By altering the thickness of the electrode, the real and imaginary parts of the impedance can be tailored for a specific signal transduction, an approach that has been used to optimize both electrode recording ${ }^{248-251}$ and stimulation ${ }^{251,252}$ of neuronal cells operating up to and beyond $1 \mathrm{kHz}$ (which corresponds to the approximate speed of synaptic signalling), and to control the development ${ }^{253}$ of, and differentiation ${ }^{254}$ into, neural cells. More recent advances in this area include short-term and long-term evaluation of PEDOT-based microelectrodes for chronic neural prostheses and therapy applications ${ }^{255}, 256$.

Mixed ion-electron conductivity is fundamental to the mode of operation of OECTs ${ }^{222}$. These devices ${ }^{257}$, especially those based on PEDOT:PSS, are heavily explored in bioelectronics applications such as sensing ${ }^{258-260}$, controlling and/or monitoring cell growth and tissue formation $^{261-263}$, neuro-recording 223,264 and circuit applications ${ }^{265}$ of relevance for OS-cell interfacing.

In addition to impedance matching, volumetric organic electronic surfaces also provide a better morphology than flat surfaces for bio-interfacing as cell networks and tissues typically grow and are configured in 3D. Electropolymerization generates polymers with inherently open 
structures and has been explored as an approach to achieve highly porous polymers for neurointerfacing ${ }^{266}$; even dendritic and fibrillar-structured interfaces can be fabricated using this approach $^{267}$ (Fig. 9d). Alone, or in combination with other approaches (such as peptide coatings), 3D microstructured and nanostructured electrodes improve neuronal attachment and reduce gliosis (that is, the response of glia cells to damage of the neuronal system) ${ }^{267}$. Electropolymerization, however, typically results in surfaces with a wide distribution of pore sizes and varied morphology. Increased control over the size distribution of fibrillar and porous structures within the organic electronic material can lead to more defined and overall improved OS-bio interfacing. One approach in this regard is to form a composite of carbon nanotubes with PEDOT, a strategy that results in highly porous and stable electrodes with increased signal:noise ratios while recording and stimulating cardiac electrophysiological signalling ${ }^{268}$. To further decrease the size distribution of the pores, various sacrificial templating approaches have been used. In one study, polystyrene beads (with diameters of $200 \mathrm{~nm}$ and $1 \mu \mathrm{m}$ ) were close-packed on electrodes to form 2D and 3D sacrificial templates for the formation of PPy electrodes loaded with a nerve growth factor ${ }^{269}$. PPy was electropolymerized in the presence of the nerve growth factor to form a homogenous phase encompassing the densely packed polystyrene beads. Dissolution of the polystyrene phase in tetrahydrofuran generated a wellordered porous surface with voids of $200 \mathrm{~nm}$ and $1 \mu \mathrm{m}$ (Fig. 9d). The behaviour of PC12 cells seeded on top of these electrodes indicates that electrical, chemical and structural cues have a great effect on cell responses ${ }^{269}$.

\section{[H2] Biosensors based on electric double layers.}

In contrast to electropolymerized PPy and PEDOT, which form porous 3D structured films, many other organic electronic polymers, such as spin-coated P3HT (a p-type semiconductor), typically form dense films and are thus relatively impermeable to ions. When these dense films are applied in biological or electrolytic media, coupling between electronic charges and ions occurs only along the planar OS-electrolyte interface, forming and EDL (Fig. 9e). This EDL, composed of negative ions and holes (or positive ions and electrons for an ntype OS) is crucial in EGOFETs ${ }^{38,41,270,271}$. A key feature of this interface is the direct coupling of ions from the electrolyte with the holes or electrons of the OS as they are not separated by an oxide layer, which is the case when inorganic semiconductors are interfaced with electrolytes and living cells. This direct coupling enables various reactions, polarizations and couplings of biological systems and processes occurring along the OS-bio interface to be recorded and monitored using different types of EGOFET-based sensor.

A DNA sensor, for example, was constructed by covalently grafting probes onto a carboxypentyl-substituted polythiophene channel of an EGOFET ${ }^{272}$. The ionic strength of the analyte solution was varied to change the effective Debye length at the OS interface, highlighting the importance of the Debye length in screening negative DNA charges and affecting the signal transduction of the sensor. The difference in transfer curve, while operating in water and upon introducing a complementary target, was primarily observed as a shift in offcurrents (Fig. 9f). An alternative EGOFET approach is to locate the sensor functionality at the gate electrode, which can then be remotely positioned with respect to the transistor channel, drain and source (Fig. 9g). This approach was used to construct a sensor for the detection of dopamine ${ }^{273}$. In this example, a gold gate electrode coated in a SAM, composed of cysteamine 
and 4-formylphenyl boronic acid, was immersed in aqueous solution. Selective adsorption of dopamine along the gate electrode induces a dipole along the gate-water interface that modulates the energetics of the EDL. This modulation is then transduced as a change in drain current of a transistor channel composed of P3HT, operating in the EGOFET mode (Fig. 9f). Using this general and versatile approach, devices in which the gate and/or OS surfaces have been modified with various probes, receptors, enzymes, phospholipids ${ }^{274}$ and ion-selective membranes $^{275}$ have enabled EGOFET sensing and detection of penicillin ${ }^{276}, \mathrm{pH}^{277}$, electrolyte components $^{275}$, DNA ${ }^{278}$, 1-butanol ${ }^{279}$ and blood gases ${ }^{280}$ at nanomolar to millimolar concentrations.

\section{[H2] Ion and drug delivery.}

Systems based on organic electronic materials can transport and release biologically relevant ions (for example, $\mathrm{H}^{+}, \mathrm{Na}^{+}, \mathrm{Cl}^{-}, \mathrm{K}^{+}$and $\mathrm{Ca}^{2+}$ ) and charged substances (such as neurotransmitters and hormones) (Fig. 9h). The goal of many of these systems has been to deliver small amounts of a substance to a specific location to minimize the side effects and toxicity associated with standard drug delivery methods (such as bolus injections and the oral administration of systemic doses) and to increase the efficacy of treatments. The capability of organic electronics to act as delivery vehicles is based primarily on the electrical switching of the redox states of the conducting polymers: in the oxidized state, the counterions are ionically bound within the polymer film, whereas in the neutral state, these ions are free to diffuse into a surrounding electrolyte. Thus, electronically addressing the polymer film, usually through an electrode underneath it, triggers the release (delivery) of the charged substances bound within $^{281}$. This form of controlled delivery has been explored since the mid-1980s ${ }^{282}$. Generally, PPy or a polythiophene derivative, with some exceptions ${ }^{283}$, is electropolymerized in the presence of the substance to be delivered, including neurotransmitters ${ }^{282}$, anti-inflammatory compounds $^{284,285}$, oligonucleotides ${ }^{286}$ and ATP $^{287}$. Similar work, based on the volume-actuation properties of conducting polymers ${ }^{288,289}$, was used to deliver the corticosteroid dexamethasone from drug-loaded fibres ${ }^{290}$.

A second approach to transport and release biologically relevant ions from organic electronics is based on the mixed ion-electron conductivity of these materials. In particular, the model system PEDOT:PSS exhibits electronic conductivity in the PEDOT phase and ionic conductivity in the polyelectrolyte PSS phase, which can be hydrated to further enhance ionic transport. PEDOT:PSS has been studied extensively for electrophoretic transport systems, exemplified by the organic electronic ion pump (OEIP) $)^{44,291,292}$. The original OEIPs comprised a single film of PEDOT:PSS with an electrode at either end; a region in the middle of the film is chemically overoxidized ${ }^{293}$, rendering the PEDOT phase electrically insulating but leaving the ionically conducting PSS phase intact. As PSS is a polyanion, this central region is thus a cation-exchange membrane ${ }^{294}$, enabling selective electrophoretic transport of cations (while excluding anions) between the electrolytes in contact with the PEDOT:PSS electrodes at either end. In this way, OEIPs and other such electrophoretic delivery devices exhibit two important features: first, they do not require liquid flow as only ions are transported, resulting in a diffusive delivery that poses minimal risk of damaging fragile biochemical environments; and second, a direct relationship between electrical current and ion flow can be established, enabling precise doses to be administered. Planar OEIPs have been used to deliver ions (for example, $\mathrm{H}^{+}$ 
and metal ions ${ }^{44}$ ) as well as cationic neurotransmitters ${ }^{291,292}$, such as glutamate, $\gamma$-aminobutyric acid and acetylcholine, to neurons and neuron-like cells in vitro. The cellular response to neurotransmitter-receptor binding was tracked through live imaging of the flux through $\mathrm{Ca}^{2+}$ channels by using a $\mathrm{Ca}^{2+}$-sensitive dye. As OEIPs and other such drug delivery technologies are developed for therapeutic applications it is often necessary to encapsulate the devices. Devices can be encapsulated by enclosing the electrolyte reservoirs in tubing or fluidic channels. Such devices have been demonstrated for the in vivo delivery of neurotransmitters to guinea pig cochlea ${ }^{291}$ (to modulate hearing sensitivity) and the spinal cord of rats ${ }^{45}$ (to alleviate pain), as well as for the delivery of plant hormones to root tips ${ }^{295}$ (to modulate growth). The OEIP concept has been expanded to encompass a broader array of both cation-exchange membrane and anion-exchange membrane materials, enabling selective transport of either cations or anions ${ }^{296}$. These complementary materials, analogous to p-type and n-type semiconductors, have been used to develop ionic diodes ${ }^{297,298}$, transistors ${ }^{299,} 300$ and circuits ${ }^{301}$, ${ }^{302}$, and to enable high-speed vertical OEIP structures ${ }^{303}$. More recently, implantable devices that incorporate the OEIP concept into the lid of a microfluidic channel have been demonstrated in vivo for epilepsy treatment ${ }^{304,305}$.

\section{[H1] Conclusion and future perspectives}

Organic electronics is now a mature research field, and the importance of OS interfaces has been recognized since the pioneering work on OLEDs and OPVs. Electrode interface engineering has been an important factor in the development of OLEDs, and recent advances in the understanding of free-charge generation at OS-OS heterojunctions coupled with new non-fullerene acceptor materials is driving the rapid increase in the power conversion efficiency of OPVs. The physics of energy level alignment at OS-metal and OS-OS interfaces under equilibrium conditions is now well established, and numerous computational approaches are available for the prediction and modelling of these systems depending on the type of interface (for example, the strength of interaction and the materials involved). For true predictive power and the in silico design of the ground-state properties of OS-metal and OS-OS interfaces, the main remaining challenge is probably the development of cost-effective multiscale modelling approaches to determine the properties of film formation and the resulting interfacial and bulk DOS. Arguably, an even greater challenge is the development of models for determining the properties of dynamic processes, such as charge injection and the generation of free charges from excitons via exciplexes, from the ground-state electronic structure and energy level alignment at OS-metal and OS-OS interfaces. The physical understanding of these dynamic processes is increasing rapidly, to a large extent driven by OPV research. However, establishing the relationship between dynamic processes and ground-state properties is hindered by limitations in the measurement of EAs, although developments building on recent breakthroughs in IPES instrumentation will likely lead to progress in the upcoming years.

The impact of physico-chemical properties of the insulator on the structural and energetic disorder of OSs at the OS-insulator interface is already well established, enabling these properties to be modulated to optimize OFET performance. Recent advances in flexible and stretchable electronics, however, have stimulated a revival in research efforts aimed at the development of novel OS-dielectric interfaces and combinations for OFETs with high electrical and mechanical performance. Blending various OSs with soft organic matrix materials is a 
simple approach that provides numerous opportunities for the introduction of desirable features into stretchable or flexible structures. Lowering the energy consumption by decreasing the operating voltage and increasing the operational stability of OFETs, while maintaining high mechanical stretchability and conformability of otherwise brittle polymer semiconductors, will continue to be crucial to the development of flexible, stretchable and wearable circuits. In addition, blending approaches that increase air and operational stability could lead to OSs for, as of yet, almost unexplored extreme-temperature electronics. Confinement effects could be exploited to introduce flexibility and stretchability into thermoelectric devices or for 3D synaptic-like memory functionalities.

New challenges are arising from the emerging field of organic bioelectronics and the use of ion-containing layers, such as cathode interlayers (in OPVs), gate dielectrics (in OECTs and EGOFETs) and light-emitting layers (in light-emitting electrochemical cells) in 'traditional' organic electronics. First, the ground-state electronic structure of the OS and equilibrium energy level alignment with the interfacing materials are greatly affected by ion size, charge and spatial distribution. Second, the dynamic processes in these systems involve not only the motion of electrons and holes, but also ions. The physical processes of charge transport and transfer across interfaces, as well as the timescales, differ substantially between the types of charges, making the theoretical modelling of these interfaces much more complicated. Advanced in operandi methods are required to provide more insight into the OSelectrolyte interface, including the structure of the EDL, the impact of the Donnan potential, the diffusion of (macro)molecular species under ion exchange or the swelling effect of solvents and the formation porous structures when a material is hydrated (which differs from dry porosity). The phenomenon of electron transfer at the OS-electrolyte interface and the adsorption of reactants at the OS surface are today scrutinized in electrocatalysis but have not yet been much explored to introduce new functions in organic electronics.

OS-bio interfaces are being developed to enable signal transfer from the OS surface across a cell membrane to sense or actuate functions inside the cell. Organic bioelectronic drug delivery provides a route to interacting with cells and tissues using the same signalling substances and spatiotemporal signalling patterns used by a healthy biological system. So far, expression and release of biomolecules and receptors have typically been limited to small molecules. Future organic bioelectronic interfaces could express and release larger molecules, such as peptides and proteins. Biochemical signalling technology will provide deeper insight into signalling in normal and diseased states and provide a route to new therapies. Finally, it has proved difficult to establish organic bioelectronic surfaces that promote the formation of lipid bilayers directly onto an organic electronic surface. With careful design and superior composite materials, it may be possible to establish a host surface that enables a phospholipid double-layer-OS interface for a wide range of sensors, drug screening and fundamental research.

\section{Acknowledgements}

The authors acknowledge financial support from the Swedish Government Strategic Research Area in Materials Science on Functional Materials at Linköping University (Faculty Grant SFO Mat LiU No. 2009 00971), the Wallenberg Wood Science Center, the Knut and Alice Wallenberg Foundation, the Swedish Foundation for Strategic Research, Vinnova (Digital Cellulose Center and Treesearch) and the Swedish Research Council.

Author contributions 


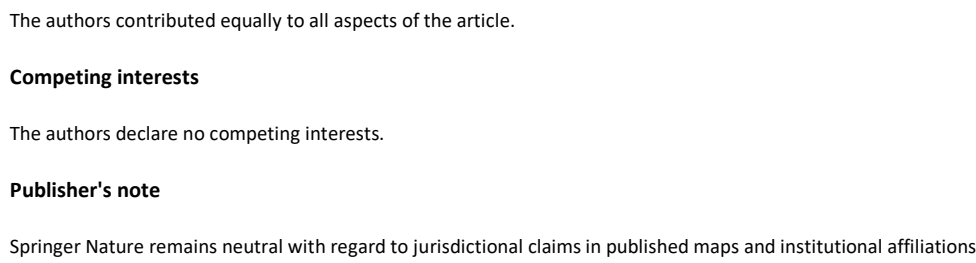

\section{References}

1.

Yoon, C.O. et al. Hopping transport in doped conducting polymers in the insulating regime near the metal-insulator boundary: polypyrrole, polyaniline and polyalkylthiophenes. Synthetic Metals 75, 229-239 (1995).

2. Lee, K. et al. Metallic transport in polyaniline. Nature 441, 65 (2006).

3. Bubnova, O. et al. Semi-metallic polymers. Nature Materials 13, 190 (2013).

4. Burroughes, J.H. et al. Light-emitting diodes based on conjugated polymers. Nature 347, 539 (1990).

5. Kulkarni, A.P., Tonzola, C.J., Babel, A. \& Jenekhe, S.A. Electron transport materials for organic light-emitting diodes. Chemistry of Materials 16, 4556-4573 (2004).

6. Blochwitz, J., Pfeiffer, M., Fritz, T. \& Leo, K. Low voltage organic light emitting diodes featuring doped phthalocyanine as hole transport material. Applied Physics Letters 73, 729-731 (1998).

7. Sun, Y. et al. Management of singlet and triplet excitons for efficient white organic light-emitting devices. Nature 440, 908 (2006).

8. Uoyama, H., Goushi, K., Shizu, K., Nomura, H. \& Adachi, C. Highly efficient organic light-emitting diodes from delayed fluorescence. Nature 492, 234 (2012).

9. Hebner, T.R., Wu, C.C., Marcy, D., Lu, M.H. \& Sturm, J.C. Ink-jet printing of doped polymers for organic light emitting devices. Applied Physics Letters 72, 519-521 (1998).

10. Han, T.-H. et al. Extremely efficient flexible organic light-emitting diodes with modified graphene anode. Nature Photonics 6, 105 (2012).

11. Sekitani, T. et al. Stretchable active-matrix organic light-emitting diode display using printable elastic conductors. Nature Materials 8, 494 (2009).

12. Reineke, S. et al. White organic light-emitting diodes with fluorescent tube efficiency. Nature 459, 234 (2009).

13. Hoppe, H. \& Sariciftci, N.S. Organic solar cells: An overview. Journal of Materials Research 19, 1924-1945 (2004).

$14 . \quad$ Darling, S.B. \& You, F. The case for organic photovoltaics. RSC Advances 3, 1763317648 (2013).

15. Meng, L. et al. Organic and solution-processed tandem solar cells with $17.3 \%$ efficiency. Science, eaat2612 (2018).

16. Azzopardi, B. et al. Economic assessment of solar electricity production from organicbased photovoltaic modules in a domestic environment. Energy \& Environmental Science 4, 37413753 (2011).

17. Diez Cabanes, V. et al. Energy Level Alignment at Interfaces Between Au (111) and Thiolated Oligophenylenes of Increasing Chain Size: Theoretical Evidence of Pinning Effects (2018).

18. Beljonne, D. et al. Electronic Processes at Organic-Organic Interfaces: Insight from Modeling and Implications for Opto-electronic Devices. Chemistry of Materials 23, 591-609 (2011). 19. Krebs, F.C. Fabrication and processing of polymer solar cells: A review of printing and coating techniques. Solar Energy Materials and Solar Cells 93, 394-412 (2009).

20. Fahlman, M. \& Salaneck, W.R. Surfaces and interfaces in polymer-based electronics. Surface Science 500, 904-922 (2002).

21. Braun, S., Salaneck, W.R. \& FahIman, M. Energy-Level Alignment at Organic/Metal and Organic/Organic Interfaces. Advanced Materials 21, 1450-1472 (2009). 
23. Dimitrakopoulos, C.D. \& Malenfant, P.R.L. Organic Thin Film Transistors for Large Area Electronics. Advanced Materials 14, 99-117 (2002).

24. Sirringhaus, H. Device Physics of Solution-Processed Organic Field-Effect Transistors. Advanced Materials 17, 2411-2425 (2005).

25. Choi, H.H., Cho, K., Frisbie, C.D., Sirringhaus, H. \& Podzorov, V. Critical assessment of charge mobility extraction in FETs. Nature Materials 17, 2 (2017).

26. Mathijssen, S.G.J. et al. Monolayer coverage and channel length set the mobility in self-assembled monolayer field-effect transistors. Nature Nanotechnology 4, 674 (2009).

27. Hulea, I.N. et al. Tunable Fröhlich polarons in organic single-crystal transistors. Nature Materials 5, 982 (2006).

28. Chua, L.-L. et al. General observation of n-type field-effect behaviour in organic semiconductors. Nature 434, 194 (2005).

29. Takeya, J. et al. Very high-mobility organic single-crystal transistors with in-crystal conduction channels. Applied Physics Letters 90, 102120 (2007).

30. Chen, H. et al. Dithiopheneindenofluorene (TIF) Semiconducting Polymers with Very High Mobility in Field-Effect Transistors. Advanced Materials 29, 1702523 (2017).

31. Wang, S. et al. Experimental evidence that short-range intermolecular aggregation is sufficient for efficient charge transport in conjugated polymers. Proceedings of the National Academy of Sciences 112, 10599 (2015).

32. Halik, M. et al. Low-voltage organic transistors with an amorphous molecular gate dielectric. Nature 431, 963 (2004).

33. Dimitrakopoulos, C.D., Purushothaman, S., Kymissis, J., Callegari, A. \& Shaw, J.M. LowVoltage Organic Transistors on Plastic Comprising High-Dielectric Constant Gate Insulators. Science 283, 822 (1999).

34. Kim, S.H. et al. Electrolyte-Gated Transistors for Organic and Printed Electronics. Advanced Materials 25, 1822-1846 (2013).

35. Naber, R.C.G., Asadi, K., Blom, P.W.M., de Leeuw, D.M. \& de Boer, B. Organic Nonvolatile Memory Devices Based on Ferroelectricity. Advanced Materials 22, 933-945 (2010).

36. Ghosh, S. \& Inganäs, O. Conducting Polymer Hydrogels as 3D Electrodes: Applications for Supercapacitors. Advanced Materials 11, 1214-1218 (1999).

37. Novák, P., Müller, K., Santhanam, K.S.V. \& Haas, O. Electrochemically Active Polymers for Rechargeable Batteries. Chemical Reviews 97, 207-282 (1997).

38. Dhoot, A.S. et al. Beyond the metal-insulator transition in polymer electrolyte gated polymer field-effect transistors. Proceedings of the National Academy of Sciences of the United States of America 103, 11834-11837 (2006).

39. Qibing, P., Gang, Y., Chi, Z., Yang, Y. \& Alan, J.H. Polymer Light-Emitting Electrochemical Cells. Science 269, 1086-1088 (1995).

40. Shin, J.H., Robinson, N.D., Xiao, S. \& Edman, L. Polymer Light-Emitting Electrochemical Cells: Doping Concentration, Emission-Zone Position, and Turn-On Time. Advanced Functional Materials 17, 1807-1813 (2007).

41. Herlogsson, L. et al. Low-voltage polymer field-effect transistors gated via a proton conductor. Advanced Materials 19, 97-101 (2007).

42. Said, E., Larsson, O., Berggren, M. \& Crispin, X. Effects of the lonic Currents in Electrolyte-gated Organic Field-Effect Transistors. Advanced Functional Materials 18, 3529-3536 (2008).

43. Cho, J.H. et al. Printable ion-gel gate dielectrics for low-voltage polymer thin-film transistors on plastic. Nature Materials 7, 900 (2008).

44. Isaksson, J. et al. Electronic control of $\mathrm{Ca} 2+$ signalling in neuronal cells using an organic electronic ion pump. Nature Materials 6, 673-679 (2007). 
45.

Jonsson, A. et al. Therapy using implanted organic bioelectronics. Science Advances 1, e1500039-e1500039 (2015).

46. Bubnova, O. et al. Optimization of the thermoelectric figure of merit in the conducting polymer poly(3,4-ethylenedioxythiophene). Nature Materials 10, 429 (2011).

47. Russ, B., Glaudell, A., Urban, J.J., Chabinyc, M.L. \& Segalman, R.A. Organic

thermoelectric materials for energy harvesting and temperature control. Nature Reviews Materials 1, 16050 (2016).

48. Rivnay, J., Owens, R.M. \& Malliaras, G.G. The Rise of Organic Bioelectronics. Chemistry of Materials 26, 679-685 (2014).

49. Simon, D.T., Gabrielsson, E.O., Tybrandt, K. \& Berggren, M. Organic Bioelectronics: Bridging the Signaling Gap between Biology and Technology. Chemical Reviews 116, 13009-13041 (2016).

50. Mitraka, E. et al. Electrocatalytic Production of Hydrogen Peroxide with Poly(3,4ethylenedioxythiophene) Electrodes. Advanced Sustainable Systems 0, 1800110 (2018).

51. Coskun, $\mathrm{H}$. et al. Biofunctionalized conductive polymers enable efficient CO\&lt;sub\&gt;2\&lt;/sub\&gt; electroreduction. Science Advances 3, e1700686 (2017).

52. Malti, A. et al. An Organic Mixed Ion-Electron Conductor for Power Electronics. Advanced Science 3, 1500305 (2016).

53. Wang, Z. et al. Surface Modified Nanocellulose Fibers Yield Conducting Polymer-Based Flexible Supercapacitors with Enhanced Capacitances. ACS Nano 9, 7563-7571 (2015).

54. Ishii, H., Sugiyama, K., Ito, E. \& Seki, K. Energy level alignment and interfacial electronic structures at organic/metal and organic/organic interfaces. Adv. Mater. 11, 972-972 (1999).

55. Lang, N.D. \& Kohn, W. Theory of Metal Surfaces: Work Function*. Phys. Rev. B 3, 1215 (1971).

56. Chen, Y.C., Cunnigham, J.E. \& Flynn, C.P. Dependence of rare-gas-adsorbate dipole moment on substrate work function. Phys. Rev. B 30, 7317 (1984).

57. Crispin, X. et al. Characterization of the Interface Dipole at Organic-Metal Interfaces. J. Am. Chem. Soc. 124, 8132-8141 (2002).

58. Osikowicz, W. et al. Energetics at Au top and bottom contacts on conjugated polymers. Appl Phys Lett 88, 193504 (2006).

59. Goiri, E., Borghetti, P., El-Sayed, A., Ortega, J.E. \& de Oteyza, D.G. Multi-Component Organic Layers on Metal Substrates. Adv. Mater. 28, 1340-1368 (2016).

60. Monti, O.L.A. Understanding Interfacial Electronic Structure and Charge Transfer: An Electrostatic Perspective. J. Phys. Chem. Lett. 3, 2342-2351 (2012).

61. van Reenen, S., Kouijzer, S., Janssen, R.A.J., Wienk, M.M. \& Kemerink, M. Origin of Work Function Modification by lonic and Amine-Based Interface Layers. Advanced Materials Interfaces 1 (2014).

62. Bao, Q.Y., Liu, X.J., Braun, S., Gao, F. \& Fahlman, M. Energetics at Doped Conjugated Polymer/Electrode Interfaces. Advanced Materials Interfaces 2 (2015).

63. Mityashin, A. et al. Unraveling the Mechanism of Molecular Doping in Organic Semiconductors. Advanced Materials 24, 1535-1539 (2012).

64. Bokdam, M., Cakir, D. \& Brocks, G. Fermi level pinning by integer charge transfer at electrode-organic semiconductor interfaces. Appl. Phys. Lett. 98, 113303 (2011).

65. Greiner, M.T. et al. Universal energy-level alignment of molecules on metal oxides.

Nat. Mater. 11, 76-81 (2011).

66. Ley, L., Smets, Y., Pakes, C.I. \& Ristein, J. Calculating the universal energy-level alignment of organic molecules on metal oxides. Adv. Funct. Mater. 23, 794-805 (2013).

67. Oehzelt, M., Koch, N. \& Heimel, G. Organic semiconductor density of states controls the energy level alignment at electrode interfaces. Nature Communications 5 (2014).

68. Salaneck, W.R. Intermolecular relaxation energies in anthracene. Phys. Rev. Lett. 40, 60 (1978). 
69. Duhm, S. et al. Orientation-dependent ionization energies and interface dipoles in ordered molecular assemblies. Nature Materials 7, 326-332 (2008).

70. Bao, Q.Y. et al. Energy Level Bending in Ultrathin Polymer Layers Obtained through Langmuir-Shafer Deposition. Advanced Functional Materials 26, 1077-1084 (2016).

71. Lindell, L., Vahlberg, C., Uvdal, K., Fahlman, M. \& Braun, S. Self-assembled monolayer engineered interfaces: Energy levelalignment tuning through chain length and end-group polarity. Journal of Electron Spectroscopy and Related Phenomena 240, 140 (2015).

72. Kotadiya, N.B. et al. Universal strategy for Ohmic hole injection into organic semiconductors with high ionization energies. Nature Mater. 17, 329 (2018).

73. Friederich, P. et al. Rational In Silico Design of an Organic Semiconductor with Improved Electron Mobility. Advanced Materials 29 (2017).

74. Cakir, D., Bokdam, M., de Jong, M.P., Fahlman, M. \& Brocks, G. Modeling charge transfer at organic donor-acceptor semiconductor interfaces. Appl. Phys. Lett. 100, 203302 (2012). 75. Yang, J.P., Bussolotti, F., Kera, S. \& Ueno, N. Origin and role of gap states in organic semiconductor studied by UPS: as the nature of organic molecular crystals. Journal of Physics $D$ Applied Physics 50 (2017).

76. Verlaak, S. et al. Electronic Structure and Geminate Pair Energetics at Organic-Organic Interfaces: The Case of Pentacene/C-60 Heterojunctions. Advanced Functional Materials 19, 38093814 (2009).

77. Mendez, H. et al. Doping of Organic Semiconductors: Impact of Dopant Strength and Electronic Coupling. Angewandte Chemie-International Edition 52, $7751-7755$ (2013).

78. Mendez, H. et al. Charge-transfer crystallites as molecular electrical dopants. Nature Communications 6 (2015).

79. Zhang, Q. et al. Ground-state charge transfer for NIR absorption with donor/acceptor molecules: interactions mediated via energetics and orbital symmetries. Journal of Materials Chemistry C 5, 275-281 (2017).

80. Breuer, T., Karthauser, A. \& Witte, G. Effects of Molecular Orientation in AcceptorDonor Interfaces between Pentacene and C-60 and Diels-Alder Adduct Formation at the Molecular Interface. Advanced Materials Interfaces 3 (2016).

81. Braun, S., Liu, X., Salaneck, W.R. \& Fahlman, M. Fermi level equilibrium at donoracceptor interfaces in multi-layered thin film stack of TTF and TCNQ. Org. Electron. 11, 212-217 (2010).

82. Brocks, G., Cakir, D., Bokdam, M., de Jong, M.P. \& Fahlman, M. Charge equilibration and potential steps in organic semiconductor multilayers. Org. Electron. 13, 1793-1801 (2012). 83. Oehzelt, M., Akaike, K., Koch, N. \& Heimel, G. Energy-level alignment at organic heterointerfaces. Science Advances 1 (2015).

84. Liu, X.K. et al. Nearly $100 \%$ Triplet Harvesting in Conventional Fluorescent DopantBased Organic Light-Emitting Devices Through Energy Transfer from Exciplex. Adv. Mater. 27, 2025 ( 2015).

85. Vandewal, K., Tvingstedt, K., Gadisa, A., Inganas, O. \& Manca, J.V. On the origin of the open-circuit voltage of polymer-fullerene solar cells. Nature Materials 8, 904-909 (2009).

86. Guan, Z.Q. et al. Charge-Transfer State Energy and Its Relationship with Open-Circuit Voltage in an Organic Photovoltaic Device. Journal of Physical Chemistry C 120, 14059-14068 (2016). 87. Yang, Q.D. et al. Probing the Energy Level Alignment and the Correlation with OpenCircuit Voltage in Solution-Processed Polymeric Bulk Heterojunction Photovoltaic Devices. Acs Applied Materials \& Interfaces 8, 7283-7290 (2016).

88. Tada, A., Geng, Y.F., Wei, Q.S., Hashimoto, K. \& Tajima, K. Tailoring organic heterojunction interfaces in bilayer polymer photovoltaic devices. Nature Materials 10, 450-455 (2011).

89. Aarnio, H. et al. Spontaneous Charge Transfer and Dipole Formation at the Interface Between P3HT and PCBM. Adv. Energy Mater. 1, 792 (2011). 
90.

Arkhipov, V.I., Heremans, P. \& Bassler, H. Why is exciton dissociation so efficient at the interface between a conjugated polymer and an electron acceptor? Appl. Phys. Lett. 82, 4605-4607 (2003).

91. Bao, Q.Y. et al. Trap-Assisted Recombination via Integer Charge Transfer States in Organic Bulk Heterojunction Photovoltaics. Advanced Functional Materials 24, 6309-6316 (2014). 92. Proudian, A.P. et al. Effect of Diels-Alder Reaction in C-60-Tetracene Photovoltaic Devices. Nano Letters 16, 6086-6091 (2016).

93. Liu, J. et al. Fast charge separation in a non-fullerene organic solar cell with a small driving force. Nature Energy 1 (2016).

94. Benduhn, J. et al. Intrinsic non-radiative voltage losses in fullerene-based organic solar cells. Nature Energy 2 (2017).

95. Jakowetz, A.C. et al. Visualizing excitations at buried heterojunctions in organic semiconductor blends. Nature Materials 16, 551-+ (2017).

96. Weiss, L.R. et al. Strongly exchange-coupled triplet pairs in an organic semiconductor. Nature Physics 13, 176-181 (2017).

97. Qian, D.P. et al. Design rules for minimizing voltage losses in high-efficiency organic solar cells. Nature Materials 17, 703-709 (2018).

98. Menke, S.M. et al. Order enables efficient electron-hole separation at an organic heterojunction with a small energy loss. Nature Communications 9 (2018).

99. Yoshida, H. Principle and application of low energy inverse photoemission spectroscopy: A new method for measuring unoccupied states of organic semiconductors. Journal of Electron Spectroscopy and Related Phenomena 204, 116-124 (2015).

100. Sirringhaus, H. 25th Anniversary Article: Organic Field-Effect Transistors: The Path Beyond Amorphous Silicon. Advanced Materials 26, 1319-1335 (2014).

101. Lee, E.K., Lee, M.Y., Park, C.H., Lee, H.R. \& Oh, J.H. Toward Environmentally Robust Organic Electronics: Approaches and Applications. Advanced Materials 29, 1703638 (2017).

102. Don Park, Y., Lim, J.A., Lee, H.S. \& Cho, K. Interface engineering in organic transistors. Materials Today 10, 46-54 (2007).

103. Di, C.-a., Liu, Y., Yu, G. \& Zhu, D. Interface Engineering: An Effective Approach toward High-Performance Organic Field-Effect Transistors. Accounts of Chemical Research 42, 1573-1583 (2009).

104. Patel, B.B. \& Diao, Y. Multiscale assembly of solution-processed organic electronics: the critical roles of confinement, fluid flow, and interfaces. Nanotechnology 29, 044004 (2018). 105. Fabiano, S. \& Pignataro, B. Selecting speed-dependent pathways for a programmable nanoscale texture by wet interfaces. Chemical Society Reviews 41, 6859-6873 (2012).

106. de Gennes, P.G. Wetting: statics and dynamics. Reviews of Modern Physics 57, 827-863 (1985).

107. Diao, Y. et al. Solution coating of large-area organic semiconductor thin films with aligned single-crystalline domains. Nature Materials 12, 665 (2013).

108. Lee, S.S. et al. Guiding Crystallization around Bends and Sharp Corners. Advanced Materials 24, 2692-2698 (2012).

109. Niazi, M.R. et al. Contact-Induced Nucleation in High-Performance Bottom-Contact Organic Thin Film Transistors Manufactured by Large-Area Compatible Solution Processing. Advanced Functional Materials 26, 2371-2378 (2016).

110. Kline, R.J. et al. Controlling the Microstructure of Solution-Processable Small Molecules in Thin-Film Transistors through Substrate Chemistry. Chemistry of Materials 23, 11941203 (2011).

111. Casalini, S., Bortolotti, C.A., Leonardi, F. \& Biscarini, F. Self-assembled monolayers in organic electronics. Chemical Society Reviews 46, 40-71 (2017).

112. Joseph Kline, R., McGehee, M.D. \& Toney, M.F. Highly oriented crystals at the buried interface in polythiophene thin-film transistors. Nature Materials 5, 222 (2006). 
113.

Kim, D.H. et al. Enhancement of Field-Effect Mobility Due to Surface-Mediated Molecular Ordering in Regioregular Polythiophene Thin Film Transistors. Advanced Functional Materials 15, 77-82 (2005).

114. Fritz, S.E., Kelley, T.W. \& Frisbie, C.D. Effect of Dielectric Roughness on Performance of Pentacene TFTs and Restoration of Performance with a Polymeric Smoothing Layer. The Journal of Physical Chemistry B 109, 10574-10577 (2005).

115. Yang, S.Y., Shin, K. \& Park, C.E. The Effect of Gate-Dielectric Surface Energy on Pentacene Morphology and Organic Field-Effect Transistor Characteristics. Advanced Functional Materials 15, 1806-1814 (2005).

116. Zhang, L., Colella, N.S., Cherniawski, B.P., Mannsfeld, S.C.B. \& Briseno, A.L. Oligothiophene Semiconductors: Synthesis, Characterization, and Applications for Organic Devices. ACS Applied Materials \& Interfaces 6, 5327-5343 (2014).

117. Mas-Torrent, M. \& Rovira, C. Role of Molecular Order and Solid-State Structure in Organic Field-Effect Transistors. Chemical Reviews 111, 4833-4856 (2011).

118. Jones, A.O.F., Chattopadhyay, B., Geerts, Y.H. \& Resel, R. Substrate-Induced and ThinFilm Phases: Polymorphism of Organic Materials on Surfaces. Advanced Functional Materials 26, 2233-2255 (2016).

119. Walter, S.R. et al. In-Situ Probe of Gate Dielectric-Semiconductor Interfacial Order in Organic Transistors: Origin and Control of Large Performance Sensitivities. Journal of the American Chemical Society 134, 11726-11733 (2012).

120. Jung, Y. et al. The Effect of Interfacial Roughness on the Thin Film Morphology and Charge Transport of High-Performance Polythiophenes. Advanced Functional Materials 18, 742-750 (2008).

121. Li, M. et al. Impact of Interfacial Microstructure on Charge Carrier Transport in Solution-Processed Conjugated Polymer Field-Effect Transistors. Advanced Materials 28, 2245-2252 (2016).

122. Tseng, H.-R. et al. High-Mobility Field-Effect Transistors Fabricated with Macroscopic Aligned Semiconducting Polymers. Advanced Materials 26, 2993-2998 (2014).

123. Lee, B.H. et al. Flexible Organic Transistors with Controlled Nanomorphology. Nano Letters 16, 314-319 (2016).

124. Hartmann, L. et al. 2D Versus 3D Crystalline Order in Thin Films of Regioregular Poly(3hexylthiophene) Oriented by Mechanical Rubbing and Epitaxy. Advanced Functional Materials 21, 4047-4057 (2011).

125. Kim, C., Facchetti, A. \& Marks, T.J. Polymer Gate Dielectric Surface Viscoelasticity Modulates Pentacene Transistor Performance. Science 318, 76-80 (2007).

126. Mohammadi, E. et al. Dynamic-template-directed multiscale assembly for large-area coating of highly-aligned conjugated polymer thin films. Nature Communications 8, 16070 (2017). 127. Baeg, K.-J., Facchetti, A. \& Noh, Y.-Y. Effects of gate dielectrics and their solvents on characteristics of solution-processed $\mathrm{N}$-channel polymer field-effect transistors. Journal of Materials Chemistry 22, 21138-21143 (2012).

128. Gaikwad, A.M. et al. Identifying orthogonal solvents for solution processed organic transistors. Organic Electronics 30, 18-29 (2016).

129. Sun, X., Di, C.-a. \& Liu, Y. Engineering of the dielectric-semiconductor interface in organic field-effect transistors. Journal of Materials Chemistry 20, 2599-2611 (2010).

130. Tsao, H.N. \& Müllen, K. Improving polymer transistor performance via morphology control. Chemical Society Reviews 39, 2372-2386 (2010).

131. Wang, C., Dong, H., Jiang, L. \& Hu, W. Organic semiconductor crystals. Chemical Society Reviews 47, 422-500 (2018).

132. Wang, B. et al. High-k Gate Dielectrics for Emerging Flexible and Stretchable Electronics. Chemical Reviews 118, 5690-5754 (2018).

133. Nketia-Yawson, B. \& Noh, Y.-Y. Recent Progress on High-Capacitance Polymer Gate Dielectrics for Flexible Low-Voltage Transistors. Advanced Functional Materials 28, 1802201 (2018). 
134. Veres, J., Ogier, S.D., Leeming, S.W., Cupertino, D.C. \& Mohialdin Khaffaf, S. Low-k Insulators as the Choice of Dielectrics in Organic Field-Effect Transistors. Adv. Funct. Mater. 13, 199204 (2003).

135. Ortiz, R.P., Facchetti, A. \& Marks, T.J. High-k Organic, Inorganic, and Hybrid Dielectrics for Low-Voltage Organic Field-Effect Transistors. Chemical Reviews 110, 205-239 (2010).

136. Richards, T., Bird, M. \& Sirringhaus, H. A quantitative analytical model for static dipolar disorder broadening of the density of states at organic heterointerfaces. The Journal of Chemical Physics 128, 234905 (2008).

137. Zhao, N. et al. Polaron Localization at Interfaces in High-Mobility Microcrystalline Conjugated Polymers. Advanced Materials 21, 3759-3763 (2009).

138. Veres, J., Ogier, S., Lloyd, G. \& de Leeuw, D. Gate Insulators in Organic Field-Effect Transistors. Chemistry of Materials 16, 4543-4555 (2004).

139. Germs, W.C., Guo, K., Janssen, R.A.J. \& Kemerink, M. Unusual Thermoelectric Behavior Indicating a Hopping to Bandlike Transport Transition in Pentacene. Physical Review Letters 109, 016601 (2012).

140. Minder, N.A., Ono, S., Chen, Z.H., Facchetti, A. \& Morpurgo, A.F. Band-Like Electron Transport in Organic Transistors and Implication of the Molecular Structure for Performance Optimization. Advanced Materials 24, 503-+ (2012).

141. Sirringhaus, H. Reliability of Organic Field-Effect Transistors. Advanced Materials 21, 3859-3873 (2009).

142. Yoon, M.-H., Kim, C., Facchetti, A. \& Marks, T.J. Gate Dielectric Chemical Structure-Organic Field-Effect Transistor Performance Correlations for Electron, Hole, and Ambipolar Organic Semiconductors. Journal of the American Chemical Society 128, 12851-12869 (2006).

143. Chua, L.L. et al. General observation of n-type field-effect behaviour in organic semiconductors. Nature 434, 194-199 (2005).

144. Kim, C. et al. Printable Cross-Linked Polymer Blend Dielectrics. Design Strategies, Synthesis, Microstructures, and Electrical Properties, with Organic Field-Effect Transistors as Testbeds. Journal of the American Chemical Society 130, 6867-6878 (2008).

145. Wang, A., Kymissis, I., Bulovic, V. \& Akinwande, A.I. Engineering density of semiconductor-dielectric interface states to modulate threshold voltage in OFETs. IEEE Transactions on Electron Devices 53, 9-13 (2006).

146. Lee, W.H., Choi, H.H., Kim, D.H. \& Cho, K. 25th Anniversary Article: Microstructure Dependent Bias Stability of Organic Transistors. Advanced Materials 26, 1660-1680 (2014).

147. Mathijssen, S.G.J. et al. Dynamics of Threshold Voltage Shifts in Organic and Amorphous Silicon Field-Effect Transistors. Advanced Materials 19, 2785-2789 (2007).

148. Goldmann, C., Gundlach, D.J. \& Batlogg, B. Evidence of water-related discrete trap state formation in pentacene single-crystal field-effect transistors. Applied Physics Letters 88, 063501 (2006).

149. Ng, T.N., Marohn, J.A. \& Chabinyc, M.L. Comparing the kinetics of bias stress in organic field-effect transistors with different dielectric interfaces. Journal of Applied Physics 100, 084505 (2006).

150. Mathijssen, S.G.J. et al. Revealing Buried Interfaces to Understand the Origins of Threshold Voltage Shifts in Organic Field-Effect Transistors. Advanced Materials 22, 5105-5109 (2010).

151. Gholamrezaie, F. et al. Charge Trapping by Self-Assembled Monolayers as the Origin of the Threshold Voltage Shift in Organic Field-Effect Transistors. Small 8, 241-245 (2012).

152. Sandberg, H.G.O., Bäcklund, T.G., Österbacka, R. \& Stubb, H. High-Performance AllPolymer Transistor Utilizing a Hygroscopic Insulator. Advanced Materials 16, 1112-1115 (2004). 153. Lee, C.A. et al. Hysteresis mechanism and reduction method in the bottom-contact pentacene thin-film transistors with cross-linked poly(vinyl alcohol) gate insulator. Applied Physics Letters 88, 252102 (2006). 
154.

Sinno, H., Fabiano, S., Crispin, X., Berggren, M. \& Engquist, I. Bias stress effect in polyelectrolyte-gated organic field-effect transistors. Applied Physics Letters 102, 5 (2013).

155. Kalb, W.L., Mathis, T., Haas, S., Stassen, A.F. \& Batlogg, B. Organic small molecule fieldeffect transistors with Cytop $^{\text {TM }}$ gate dielectric: Eliminating gate bias stress effects. Applied Physics Letters 90, 092104 (2007).

156. Kobayashi, S. et al. Control of carrier density by self-assembled monolayers in organic field-effect transistors. Nature Materials 3, 317 (2004).

157. Pernstich, K.P. et al. Threshold voltage shift in organic field effect transistors by dipole monolayers on the gate insulator. Journal of Applied Physics 96, 6431-6438 (2004).

158. Chung, Y. et al. Controlling Electric Dipoles in Nanodielectrics and Its Applications for Enabling Air-Stable n-Channel Organic Transistors. Nano Letters 11, 1161-1165 (2011).

159. Boudinet, D. et al. Influence of Substrate Surface Chemistry on the Performance of Top-Gate Organic Thin-Film Transistors. Journal of the American Chemical Society 133, 9968-9971 (2011).

160. Baeg, K.J. et al. Remarkable Enhancement of Hole Transport in Top-Gated N-Type Polymer Field-Effect Transistors by a High-k Dielectric for Ambipolar Electronic Circuits. Adv. Mater.

24, 5433-5439 (2012).

161. Huang, C., Katz, H.E. \& West, J.E. Solution-Processed Organic Field-Effect Transistors and Unipolar Inverters Using Self-Assembled Interface Dipoles on Gate Dielectrics. Langmuir 23, 13223-13231 (2007).

162. Guo, Y., Yu, G. \& Liu, Y. Functional Organic Field-Effect Transistors. Advanced Materials 22, 4427-4447 (2010).

163. Zhang, H. et al. Interface Engineering of Semiconductor/Dielectric Heterojunctions toward Functional Organic Thin-Film Transistors. Nano Letters 11, $4939-4946$ (2011).

164. Baeg, K.-J. et al. Organic Non-Volatile Memory Based on Pentacene Field-Effect Transistors Using a Polymeric Gate Electret. Advanced Materials 18, 3179-3183 (2006).

165. Baeg, K.-J., Noh, Y.-Y., Ghim, J., Lim, B. \& Kim, D.-Y. Polarity Effects of Polymer Gate Electrets on Non-Volatile Organic Field-Effect Transistor Memory. Advanced Functional Materials 18, 3678-3685 (2008).

166. Heremans, P. et al. Polymer and Organic Nonvolatile Memory Devices. Chemistry of Materials 23, 341-358 (2011).

167. Naber, R.C.G., Asadi, K., Blom, P.W.M., de Leeuw, D.M. \& de Boer, B. Organic Nonvolatile Memory Devices Based on Ferroelectricity. Adv. Mater. 22 (2010).

$168 . \quad$ Naber, R.C.G. et al. High-performance solution-processed polymer ferroelectric fieldeffect transistors. Nat. Mater. 4, 243-248 (2005).

169. Naber, R.C.G., Blom, P.W.M., Gelinck, G.H., Marsman, A.W. \& de Leeuw, D.M. An organic field-effect transistor with programmable polarity. Adv. Mater. 17 (2005).

170. Fabiano, S. et al. Selective Remanent Ambipolar Charge Transport in Polymeric FieldEffect Transistors For High-Performance Logic Circuits Fabricated in Ambient. Adv. Mater. 26, 74387443 (2014).

171. Fabiano, S., Crispin, X. \& Berggren, M. Ferroelectric Polarization Induces Electric Double Layer Bistability in Electrolyte-Gated Field-Effect Transistors. ACS Appl. Mater. Interfaces 6 , 438-442 (2014).

172. Fabiano, S. et al. Ferroelectric polarization induces electronic nonlinearity in ion-doped conducting polymers. Science Advances 3, e1700345 (2017).

173. Wang, H. et al. A Ferroelectric/Electrochemical Modulated Organic Synapse for Ultraflexible, Artificial Visual-Perception System. Advanced Materials 30, 1803961 (2018).

174. Goffri, S. et al. Multicomponent semiconducting polymer systems with low crystallization-induced percolation threshold. Nat. Mater. 5, 950-956 (2006).

175. Lamont, C.A. et al. Tuning the viscosity of halogen free bulk heterojunction inks for inkjet printed organic solar cells. Organic Electronics 17, 107-114 (2015). 
176.

Scaccabarozzi, A.D. \& Stingelin, N. Semiconducting:insulating polymer blends for optoelectronic applications-a review of recent advances. Journal of Materials Chemistry A 2, 1081810824 (2014).

177. Arias, A.C., Endicott, F. \& Street, R.A. Surface-induced self-encapsulation of polymer thin-film transistors. Advanced Materials 18, 2900-+ (2006).

178. Hellmann, C. et al. Controlling the Interaction of Light with Polymer Semiconductors. Advanced Materials 25, 4906-4911 (2013).

179. Wang, S. et al. Experimental evidence that short-range intermolecular aggregation is sufficient for efficient charge transport in conjugated polymers. Proc. Natl. Acad. Sci. U.S.A. 112, 10599-10604 (2015).

180. del Pozo, F.G. et al. Single Crystal-Like Performance in Solution-Coated Thin-Film Organic Field-Effect Transistors. Advanced Functional Materials 26, 2379-2386 (2016).

181. Abbaszadeh, D. et al. Elimination of charge carrier trapping in diluted semiconductors. Nature Materials 15, 628 (2016).

182. Si, L., Massa, M.V., Dalnoki-Veress, K., Brown, H.R. \& Jones, R.A.L. Chain Entanglement in Thin Freestanding Polymer Films. Physical Review Letters 94, 127801 (2005).

183. Shin, K. et al. Crystalline Structures, Melting, and Crystallization of Linear Polyethylene in Cylindrical Nanopores. Macromolecules 40, 6617-6623 (2007).

184. Xu, J. et al. Highly stretchable polymer semiconductor films through the nanoconfinement effect. Science 355, 59-64 (2017).

185. Gumyusenge, A. et al. Semiconducting polymer blends that exhibit stable charge transport at high temperatures. Science 362, 1131-1134 (2018).

186. Kumar, A., Baklar, M.A., Scott, K., Kreouzis, T. \& Stingelin-Stutzmann, N. Efficient, Stable Bulk Charge Transport in Crystalline/Crystalline Semiconductor-Insulator Blends. Advanced Materials 21, 4447-4451 (2009).

187. Lu, G. et al. Moderate doping leads to high performance of semiconductor/insulator polymer blend transistors. Nat. Commun. 4, 1588 (2013).

188. $\quad \mathrm{Yu}, \mathrm{X}$. et al. High-Performance Field-Effect Transistors Based on Polystyrene-b-Poly(3hexylthiophene) Diblock Copolymers. ACS Nano 5, 3559-3567 (2011).

189. Hamilton, R. et al. High-Performance Polymer-Small Molecule Blend Organic Transistors. Advanced Materials 21, 1166-1171 (2009).

190. Sun, Z. et al. PS-b-P3HT Copolymers as P3HT/PCBM Interfacial Compatibilizers for High Efficiency Photovoltaics. Advanced Materials 23, 5529-5535 (2011).

191. Sauvé, G. \& McCullough, R.D. High Field-Effect Mobilities for Diblock Copolymers of Poly(3-hexylthiophene) and Poly(methyl acrylate). Advanced Materials 19, 1822-1825 (2007).

192. Diaz, A.F., Kanazawa, K.K. \& Gardini, G.P. Electrochemical polymerization of pyrrole. Journal of the Chemical Society, Chemical Communications, 635-636 (1979).

193. Diaz, A.F. \& Logan, J.A. Electroactive polyaniline films. Journal of Electroanalytical Chemistry and Interfacial Electrochemistry 111, 111-114 (1980).

194. Xuan, Y. et al. Thermoelectric properties of conducting polymers: The case of poly(3hexylthiophene). Physical Review B 82, 115454 (2010).

195. Donnan, F.G. Theory of membrane equilibria and membrane potentials in the presence of non-dialysing electrolytes. A contribution to physical-chemical physiology. Journal of Membrane Science 100, 45-55 (1995).

196. Mafé, S., Manzanares, J.A. \& Reiss, H. Donnan phenomena in membranes with charge due to ion adsorption. Effects of the interaction between adsorbed charged groups. The Journal of Chemical Physics 98, 2325-2331 (1993).

197. Scotto, J., Florit, M.I. \& Posadas, D. The effect of membrane equilibrium on the behaviour of electrochemically active polymers. Journal of Electroanalytical Chemistry 774, 42-50 (2016).

198. Rubinson, J.F. \& Kayinamura, Y.P. Charge transport in conducting polymers: insights from impedance spectroscopy. Chemical Society Reviews 38, 3339-3347 (2009). 
199.

Pajkossy, T. \& Jurczakowski, R. Electrochemical impedance spectroscopy in interfacial studies. Current Opinion in Electrochemistry 1, 53-58 (2017).

200. Stavrinidou, E., Sessolo, M., Winther-Jensen, B., Sanaur, S. \& Malliaras, G.G. A physical interpretation of impedance at conducting polymer/electrolyte junctions. AIP Advances 4, 017127 (2014).

201. Johansson, T., Persson, N.-K. \& Inganäs, O. Moving Redox Fronts in Conjugated Polymers Studies from Lateral Electrochemistry in Polythiophenes. Journal of The Electrochemical Society 151, E119-E124 (2004).

202. Yao, L., Rahmanudin, A., Guijarro, N. \& Sivula, K. Organic Semiconductor Based Devices for Solar Water Splitting. Advanced Energy Materials 8, 1802585 (2018).

203. Argun, A.A. et al. Multicolored Electrochromism in Polymers: Structures and Devices. Chemistry of Materials 16, 4401-4412 (2004).

204. Erlandsson, P.G. \& Robinson, N.D. Electrolysis-reducing electrodes for electrokinetic devices. ELECTROPHORESIS 32, 784-790 (2011).

205. Jager, E.W.H., Smela, E., Inganäs, O. \& Lundström, I. Polypyrrole micro actuators. Synthetic Metals 102, 1309-1310 (1999).

206. Mirfakhrai, T., Madden, J.D.W. \& Baughman, R.H. Polymer artificial muscles. Materials Today 10, 30-38 (2007).

207. Orata, D. \& Buttry, D.A. Determination of ion populations and solvent content as functions of redox state and $\mathrm{pH}$ in polyaniline. Journal of the American Chemical Society 109, 35743581 (1987).

208. Smela, E. \& Gadegaard, N. Volume Change in Polypyrrole Studied by Atomic Force Microscopy. The Journal of Physical Chemistry B 105, 9395-9405 (2001).

209. Giridharagopal, R. et al. Electrochemical strain microscopy probes morphologyinduced variations in ion uptake and performance in organic electrochemical transistors. Nature Materials 16, 737 (2017).

210. Döbbelin, M. et al. A new approach to hydrophobic and water-resistant poly(3,4ethylenedioxythiophene):poly(styrenesulfonate) films using ionic liquids. Journal of Materials Chemistry 18, 5354-5358 (2008).

211. Lin, P., Yan, F. \& Chan, H.L.W. Improvement of the Tunable Wettability Property of Poly(3-alkylthiophene) Films. Langmuir 25, 7465-7470 (2009).

212. Darmanin, T. \& Guittard, F. Wettability of conducting polymers: From superhydrophilicity to superoleophobicity. Progress in Polymer Science 39, 656-682 (2014).

213. Robinson, L., Hentzell, A., Robinson, N.D., Isaksson, J. \& Berggren, M. Electrochemical wettability switches gate aqueous liquids in microfluidic systems. Lab on a Chip 6, 1277-1278 (2006). 214. Halldorsson, J.A., Little, S.J., Diamond, D., Spinks, G. \& Wallace, G. Controlled Transport of Droplets Using Conducting Polymers. Langmuir 25, 11137-11141 (2009).

215. DeLongchamp, D.M. \& Hammond, P.T. High-Contrast Electrochromism and Controllable Dissolution of Assembled Prussian Blue/Polymer Nanocomposites. Advanced Functional Materials 14, 224-232 (2004).

216. Persson, K.M. et al. Electronic Control over Detachment of a Self-Doped Water-Soluble Conjugated Polyelectrolyte. Langmuir 30, 6257-6266 (2014).

217. Inzelt, G., Pineri, M., Schultze, J.W. \& Vorotyntsev, M.A. Electron and proton conducting polymers: recent developments and prospects. Electrochimica Acta 45, 2403-2421 (2000).

218. Volkov, A.V. et al. Understanding the Capacitance of PEDOT:PSS. Advanced Functional Materials 27 (2017).

219. Gogotsi, Y. \& Penner, R.M. Energy Storage in Nanomaterials - Capacitive, Pseudocapacitive, or Battery-like? ACS Nano 12, 2081-2083 (2018).

220. Chen, G.Z. Supercapacitor and supercapattery as emerging electrochemical energy stores. International Materials Reviews 62, 173-202 (2017). 

of a solid-state microelectrochemical transistor based on poly(3-methylthiophene). Journal of the American Chemical Society 109, 2197-2199 (1987).

222. Rivnay, J. et al. Organic electrochemical transistors. Nature Reviews Materials 3 (2018).

223. Rivnay, J. et al. High-performance transistors for bioelectronics through tuning of channel thickness. Science Advances 1 (2015).

224. Giovannitti, A. et al. Controlling the mode of operation of organic transistors through side-chain engineering. Proceedings of the National Academy of Sciences 113, 12017-12022 (2016). 225. Sun, H. et al. Complementary Logic Circuits Based on High-Performance n-Type Organic Electrochemical Transistors. Advanced Materials 30, 1704916 (2018).

226. Herlogsson, L., Crispin, X., Tierney, S. \& Berggren, M. Polyelectrolyte-Gated Organic Complementary Circuits Operating at Low Power and Voltage. Advanced Materials 23, 4684-4689 (2011).

227. Larsson, O., Said, E., Berggren, M. \& Crispin, X. Insulator Polarization Mechanisms in Polyelectrolyte-Gated Organic Field-Effect Transistors. Advanced Functional Materials 19, 3334-3341 (2009).

228. Xie, W. \& Frisbie, C.D. Organic Electrical Double Layer Transistors Based on Rubrene Single Crystals: Examining Transport at High Surface Charge Densities above $1013 \mathrm{~cm}-2$. The Journal of Physical Chemistry C 115, 14360-14368 (2011).

229. Lee, J. et al. Ion Gel-Gated Polymer Thin-Film Transistors: Operating Mechanism and Characterization of Gate Dielectric Capacitance, Switching Speed, and Stability. The Journal of Physical Chemistry C 113, 8972-8981 (2009).

230. Laiho, A., Herlogsson, L., Forchheimer, R., Crispin, X. \& Berggren, M. Controlling the dimensionality of charge transport in organic thin-film transistors. Proceedings of the National Academy of Sciences 108, 15069-15073 (2011).

$231 . \quad$ Lin, F. \& Lonergan, M.C. Gate electrode processes in an electrolyte-gated transistor: Non-Faradaically versus Faradaically coupled conductivity modulation of a polyacetylene ionomer. Applied Physics Letters 88, 133507 (2006).

232. Tarabella, G. et al. Effect of the gate electrode on the response of organic electrochemical transistors. Applied Physics Letters 97, 123304 (2010).

233. Suda, M. et al. N-Type Superconductivity in an Organic Mott Insulator Induced by Light-Driven Electron-Doping. Advanced Materials 29, 1606833 (2017).

234. Williams, R.L. \& Doherty, P.J. A preliminary assessment of poly(pyrrole) in nerve guide studies. Journal of Materials Science: Materials in Medicine 5, 429-433 (1994).

235. Wong, J.Y., Langer, R. \& Ingber, D.E. Electrically conducting polymers can noninvasively control the shape and growth of mammalian cells. Proceedings of the National Academy of Sciences of the United States of America 91, 3201-3204 (1994).

236. Cui, X. et al. Surface modification of neural recording electrodes with conducting polymer/biomolecule blends. Journal of Biomedical Materials Research 56, 261-272 (2001).

237. Asplund, M. et al. Toxicity evaluation of PEDOT/biomolecular composites intended for neural communication electrodes. Biomedical Materials 4 (2009).

238. Saltó, C. et al. Control of neural stem cell adhesion and density by an electronic polymer surface switch. Langmuir 24, 14133-14138 (2008).

239. Svennersten, K., Bolin, M.H., Jager, E.W.H., Berggren, M. \& Richter-Dahlfors, A. Electrochemical modulation of epithelia formation using conducting polymers. Biomaterials $\mathbf{3 0}$, 6257-6264 (2009).

240. Cui, X.T. \& Martin, D.C. 101-106 (2002).

241. Luo, S.C. et al. Poly(3,4-ethylenedioxythiophene) (PEDOT) nanobiointerfaces: Thin, ultrasmooth, and functionalized PEDOT films with in vitro and in vivo biocompatibility. Langmuir 24, 8071-8077 (2008). 
Xie, C. et al. Electroresponsive and cell-affinitive polydopamine/polypyrrole composite microcapsules with a dual-function of on-demand drug delivery and cell stimulation for electrical therapy. NPG Asia Materials 9 (2017).

243. Zhou, L. et al. Soft Conducting Polymer Hydrogels Cross-Linked and Doped by Tannic Acid for Spinal Cord Injury Repair. ACS Nano (2018).

244. Li, Y. \& Yu, C. RGD peptide doped polypyrrole film as a biomimetic electrode coating for impedimetric sensing of cell proliferation and cytotoxicity. Journal of Applied Biomedicine 15, 256-264 (2017).

$245 . \quad$ Du, W. et al. Improving the Compatibility of Diketopyrrolopyrrole Semiconducting Polymers for Biological Interfacing by Lysine Attachment. Chemistry of Materials 30, 6164-6172 (2018).

246. Kim, D.H., Abidian, M. \& Martin, D.C. Conducting polymers grown in hydrogel scaffolds coated on neural prosthetic devices. Journal of Biomedical Materials Research - Part A 71, 577-585 (2004).

247. Tybrandt, K., Zozoulenko, I.V. \& Berggren, M. Chemical potential-electric double layer coupling in conjugated polymer-polyelectrolyte blends. Science Advances 3 (2017).

248. Mandal, H.S. et al. Improving the performance of poly(3,4-ethylenedioxythiophene) for brain-machine interface applications. Acta Biomaterialia 10, 2446-2454 (2014).

249. Cui, X. \& Martin, D.C. Electrochemical deposition and characterization of poly(3,4ethylenedioxythiophene) on neural microelectrode arrays. Sensors and Actuators, B: Chemical 89, 92102 (2003).

250. Ludwig, K.A., Uram, J.D., Yang, J., Martin, D.C. \& Kipke, D.R. Chronic neural recordings using silicon microelectrode arrays electrochemically deposited with a poly $(3,4-$ ethylenedioxythiophene) (PEDOT) film. Journal of Neural Engineering 3, 59-70 (2006).

251. Asplund, M., von Holst, H. \& Inganäs, O. Composite biomolecule/PEDOT materials for neural electrodes. Biointerphases 3, 83-93 (2008).

252. Cui, X.T. \& Zhou, D.D. Poly (3,4-ethylenedioxythiophene) for chronic neural stimulation. IEEE Transactions on Neural Systems and Rehabilitation Engineering 15, 502-508 (2007). 253. Collazos-Castro, J.E., Polo, J.L., Hernández-Labrado, G.R., Padial-Cañete, V. \& GarcíaRama, C. Bioelectrochemical control of neural cell development on conducting polymers.

Biomaterials 31, 9244-9255 (2010).

254. Guo, W. et al. Self-Powered Electrical Stimulation for Enhancing Neural Differentiation of Mesenchymal Stem Cells on Graphene-Poly(3,4-ethylenedioxythiophene) Hybrid Microfibers. ACS Nano 10, 5086-5095 (2016).

$255 . \quad$ Schander, A. et al. 6174-6177 (2016).

256. Alves-Sampaio, A., García-Rama, C. \& Collazos-Castro, J.E. Biofunctionalized PEDOTcoated microfibers for the treatment of spinal cord injury. Biomaterials 89, 98-113 (2016).

257. Nilsson, D. et al. Bi-stable and Dynamic Current Modulation in Electrochemical Organic Transistors. Advanced Materials 14, 51-54 (2002).

258. Nilsson, D., Kugler, T., Svensson, P.O. \& Berggren, M. An all-organic sensor-transistor based on a novel electrochemical transducer concept printed electrochemical sensors on paper. Sensors and Actuators, B: Chemical 86, 193-197 (2002).

259. Gentili, D. et al. Integration of organic electrochemical transistors and immuno-affinity membranes for label-free detection of interleukin- 6 in the physiological concentration range through antibody-antigen recognition. Journal of Materials Chemistry B 6, 5400-5406 (2018).

260. Tarabella, G. et al. Liposome sensing and monitoring by organic electrochemical transistors integrated in microfluidics. Biochimica et Biophysica Acta - General Subjects 1830, 43744380 (2013).

261. Bolin, M.H. et al. Active control of epithelial cell-density gradients grown along the channel of an organic electrochemical transistor. Advanced Materials 21, 4379-4382 (2009). 

coverage and differentiation with the organic electrochemical transistor. Journal of Materials Chemistry B 3, 5971-5977 (2015).

$263 . \quad J i m i s o n$, L.H. et al. Measurement of barrier tissue integrity with an organic electrochemical transistor. Advanced Materials 24, 5919-5923 (2012).

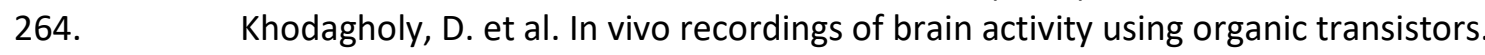
Nature Communications 4 (2013).

$265 . \quad$ Nilsson, D., Robinson, N., Berggren, M. \& Forchheimer, R. Electrochemical Logic Circuits. Advanced Materials 17, 353-358 (2005).

266. Xiao, Y., Cui, X. \& Martin, D.C. Electrochemical polymerization and properties of PEDOT/S-EDOT on neural microelectrode arrays. Journal of Electroanalytical Chemistry 573, 43-48 (2004).

267. Cui, X., Wiler, J., Dzaman, M., Altschuler, R.A. \& Martin, D.C. In vivo studies of polypyrrole/peptide coated neural probes. Biomaterials 24, 777-787 (2003).

268. Gerwig, R. et al. PEDOT-CNT composite microelectrodes for recording and electrostimulation applications: Fabrication, morphology, and electrical properties. Frontiers in Neuroengineering (2012).

269. Kang, G., Borgens, R.B. \& Cho, Y. Well-ordered porous conductive polypyrrole as a new platform for neural interfaces. Langmuir 27, 6179-6184 (2011).

270. Panzer, M.J. \& Frisbie, C.D. Polymer electrolyte-gated organic field-effect transistors: Low-voltage, high-current switches for organic electronics and testbeds for probing electrical transport at high charge carrier density. Journal of the American Chemical Society 129, 6599-6607 (2007).

271. Kergoat, L. et al. A water-gate organic field-effect transistor. Advanced Materials 22, 2565-2569 (2010).

272. Kergoat, L. et al. DNA detection with a water-gated organic field-effect transistor. Organic Electronics: physics, materials, applications 13, 1-6 (2012).

273. Casalini, S., Leonardi, F., Cramer, T. \& Biscarini, F. Organic field-effect transistor for label-free dopamine sensing. Organic Electronics: physics, materials, applications 14, 156-163 (2013). 274. Magliulo, M. et al. Electrolyte-gated organic field-effect transistor sensors based on supported biotinylated phospholipid bilayer. Advanced Materials 25, 2090-2094 (2013).

275. Schmoltner, K., Kofler, J., Klug, A. \& List-Kratochvil, E.J.W. Electrolyte-gated organic field-effect transistor for selective reversible ion detection. Advanced Materials 25, 6895-6899 (2013).

276. Buth, F., Donner, A., Stutzmann, M. \& Garrido, J.A. in Proceedings of SPIE - The International Society for Optical Engineering (2012).

277. Buth, F., Donner, A., Sachsenhauser, M., Stutzmann, M. \& Garrido, J.A. Biofunctional electrolyte-gated organic field-effect transistors. Advanced Materials 24, 4511-4517 (2012). 278. White, S.P., Dorfman, K.D. \& Frisbie, C.D. in 18th International Conference on Miniaturized Systems for Chemistry and Life Sciences, MicroTAS 2014 2047-2049 (2014).

279. Dumitru, L.M., Manoli, K., Magliulo, M., Palazzo, G. \& Torsi, L. Low-voltage solid electrolyte-gated OFETs for gas sensing applications. Microelectronics Journal 45, 1679-1683 (2014). 280. Werkmeister, F.X. \& Nickel, B.A. Fast detection of blood gases by solution gated organic field effect transistors. Organic Electronics: physics, materials, applications 39, 113-117 (2016).

281. Svirskis, D., Travas-Sejdic, J., Rodgers, A. \& Garg, S. Electrochemically controlled drug delivery based on intrinsically conducting polymers. Journal of Controlled Release 146, 6-15 (2010). 282. Zinger, B. \& Miller, L.L. Timed release of chemicals from polypyrrole films. Journal of the American Chemical Society 106, 6861-6863 (1984).

283. Lira, L.M. \& Córdoba de Torresi, S.I. Conducting polymer-hydrogel composites for electrochemical release devices: Synthesis and characterization of semi-interpenetrating polyanilinepolyacrylamide networks. Electrochemistry Communications 7, 717-723 (2005). 

pyrrole-based ion-gate membrane as studied by the EQCM. Synthetic Metals (1998).

285. Wadhwa, R., Lagenaur, C.F. \& Cui, X.T. Electrochemically controlled release of dexamethasone from conducting polymer polypyrrole coated electrode. Journal of Controlled Release 110, 531-541 (2006).

286. Wang, J. \& Jiang, M. Toward Genolelectronics: Nucleic Acid Doped Conducting Polymers. Langmuir 16, 2269-2274 (2000).

287. Pernaut, J.-M. \& Reynolds, J.R. Use of Conducting Electroactive Polymers for Drug Delivery and Sensing of Bioactive Molecules. A Redox Chemistry Approach. Journal of Physical Chemistry B 104, 11 (2000).

288. Pei, Q. \& Inganäs, O. Electrochemical muscles: Bending strips built from conjugated polymers. Synthetic Metals 57, 3718-3723 (1993).

289. Jager, E.W.H., Smela, E. \& Inganäs, O. Microfabricating conjugated polymer actuators. Science 290, 1540-1545 (2000).

290. Abidian, M.R. \& Martin, D.C. Multifunctional Nanobiomaterials for Neural Interfaces. Advanced Functional Materials 19, 573-585 (2009).

291. Simon, D.T. et al. Organic electronics for precise delivery of neurotransmitters to modulate mammalian sensory function. Nature Materials 8, 742-746 (2009).

292. Tybrandt, K. et al. Translating electronic currents to precise acetylcholine-induced neuronal signaling using an organic electrophoretic delivery device. Advanced Materials 21, 44424446 (2009).

293. Krische, B. \& Zagorska, M. Overoxidation in conducting polymers. Synthetic Metals 28, 257-262 (1989).

294. Kontturi, K., Murtomäki, L. \& Manzanares, J.A.J.A. Ionic Transport Processes: In Electrochemistry and Membrane Science. Ionic Transport Processes: In Electrochemistry and Membrane Science (2008).

$295 . \quad$ Poxson, D.J. et al. Regulating plant physiology with organic electronics. Proceedings of the National Academy of Sciences 114, 4597-4602 (2017).

296. Arbring Sjöström, T. et al. A Decade of lontronic Delivery Devices. Advanced Materials Technologies 3, 1700360 (2018).

297. Gabrielsson, E.O., Tybrandt, K. \& Berggren, M. Ion diode logics for pH control. Lab on a Chip 12, 2507-2513 (2012).

298. Gabrielsson, E.O., Tybrandt, K. \& Berggren, M. Polyphosphonium-based ion bipolar junction transistors. Biomicrofluidics 8, 64116 (2014).

$299 . \quad T y b r a n d t$, K., Larsson, K.C., Richter-Dahlfors, A. \& Berggren, M. Ion bipolar junction transistors. Proceedings of the National Academy of Sciences 107, 9929-9932 (2010).

300. Tybrandt, K., Gabrielsson, E.O. \& Berggren, M. Toward Complementary lonic Circuits: The npn Ion Bipolar Junction Transistor. Journal of the American Chemical Society 133, 10141-10145 (2011).

301. Tybrandt, K., Forchheimer, R. \& Berggren, M. Logic gates based on ion transistors. Nature Communications 3, 871 (2012).

302. Gabrielsson, E.O., Janson, P., Tybrandt, K., Simon, D.T. \& Berggren, M. A four-diode full-wave ionic current rectifier based on bipolar membranes: Overcoming the limit of electrode capacity. Advanced Materials 26, 5143-5147 (2014).

303. Jonsson, A., Arbring Sjöström, T., Tybrandt, K., Berggren, M. \& Simon, D.T. Chemical delivery array with millisecond neurotransmitter release. Science Advances 2, e1601340-e1601340 (2016).

304. Uguz, I. et al. A microfluidic ion pump for in vivo drug delivery. Advanced Materials 29, 1701217 (2017).

305. Proctor, C.M. et al. Electrophoretic drug delivery for seizure control. Science Advances 4, eaau1291 (2018). 
Fig. 1 | Structure of organic semiconductors. a | The chemical structure of a conjugated molecule (an oligothiophene); alternating single and double bonds form a conjugated path. $\mathbf{b}$ | Side view of a conjugated molecule; the $\sigma$ skeleton is planar, and the $\pi$-electronic density (originating from the interaction of the $2 p_{z}$ atomic orbitals) is perpendicular to the plane of the molecule. c | Chemical structure of a charged conjugated molecule, known as a bipolaron, in which the 2+ charge is localized on three monomers and is charge-balanced by two anions. The charge localization changes the bond length alternation. $\mathbf{d} \mid$ Electronic structure of a conjugated molecule with occupied $\pi$ and $\sigma$ orbitals and unoccupied $\pi^{*}$ and $\sigma^{*}$ orbitals. The energy of the frontier orbitals (the highest occupied molecular orbital (HOMO) and lowest unoccupied molecular orbital (LUMO)) define the ionization potential (IP) and electron affinity (EA). e | The $\pi-\pi$ interactions within organic semiconductor crystals can promote fast, thermally activated hopping transport (shown here for holes). f | Within disordered organic semiconductors, local ordering of molecules creates a percolation path. $\mathbf{g}$ | For a positively doped organic semiconductor charge-balanced with counter anions, both types of charge carriers - that is, electronic and ionic charges - are mobile. This is an example of a mixed ion-electron conductor. $E_{\mathrm{vac}}$, vacuum level.

Fig. 2 | Interfaces in organic electronic devices. Examples of organic electronic devices illustrating the roles and variety of interfaces. The left part of each panel shows the layers within the device, whereas the right part of each panel is an energy level diagram that indicates the roles of the interfaces within the device (note that the energy level diagrams are rotated by $90^{\circ}$ with respect to the device layers). a $\mid$ An organic light-emitting diode (OLED) based on undoped organic semiconductors (OSs) with OS-metal and OS-OS interfaces. b | An organic photovoltaic (OPV) solar cell based on a binary OS blend of an acceptor (A) and donor (D), featuring OS-OS and OS-metal interfaces. c | An organic field-effect transistor (OFET); this system features OS-metal and OS-electrolyte interfaces. d | A supercapacitor based on doped OSs with OS-metal and OS-electrolyte interfaces. $h v$, photon energy; $\mathrm{CB}$, conduction band; HOMO, highest occupied molecular orbital; LUMO, lowest unoccupied molecular orbital; VB, valence band; WF, work function.

Fig. 3 | Organic semiconductor-metal interfaces. a | Energy level alignment at an initially clean metal surface, showing the vacuum level (VL) shifts attributed to the chemisorbed surface layer $\left(\Delta^{\prime}\right)$ and subsequent physisorbed layers $\left(\Delta^{\prime \prime}\right) . \Delta^{\prime}$ is the sum of contributions from the pushback dipole potential step $(\Delta \mathrm{pb})$, the dipole potential step due to hybridization of the frontier molecular orbitals of the organic semiconductor and partial charge transfer $\left(\Delta_{\text {chem }}\right)$, the dipole potential step induced by absorbed molecules with an intrinsic dipole moment $\left(\Delta_{\text {intdp }}\right)$ and the double-dipole potential step $\left(\Delta_{\mathrm{ddp}}\right)$. $\Delta^{\prime \prime}$ is equal to the dipole potential step created by integer charge transfer (ICT) between a physisorbed OS layer and a metal contact $\left(\Delta_{\mathrm{ICT}}\right)$. The polaron density of states (DOS) is dependent on the distance from the interface; the bulk ionization potential (IP) and electron affinity (EA) are assigned to the edges of the polaron DOS. $E_{\text {ICT }+}$ and $E_{\text {ICT- }}$ correspond to the edges of the oxidation and reduction energy distributions at the interface, respectively. In this example, the Fermi level $\left(E_{\mathrm{F}}\right)$ is pinned to $E_{\mathrm{ICT}+}$, causing a downshift of the vacuum level. $\mathbf{b} \mid \mathrm{A} \Delta \mathrm{ddp}$ forms when the range of mobility of the two charged species in a polyelectrolyte monolayer on a metal surface differ (for example, owing to the size of the ions). When the negatively charged species are more mobile than the positively charged species, the $\Delta_{\text {ddp }}$ that forms downshifts the vacuum level (right). By contrast, when the positively charged species are more mobile than the negatively charged species, the $\Delta_{\text {ddp }}$ upshifts the vacuum level (left). c | A spacer layer can be inserted between an organic semiconductor film and a metal electrode to modify the energy level alignment. The spacer layer increases the distance between 
the charges in the organic semiconductor layer(s) and the image charges on the metal, thereby decreasing the electrostatic energy and shifting the polaron energies towards those in the bulk. $\boldsymbol{\mu}$, dipole moment; $\Phi_{\text {met }}$, metal work function; $E$, energy; $E_{\mathrm{g}}$, energy gap. Figure adapted with permission from ref. ${ }^{306}$, Wiley-VCH.

Fig. 4 | Multilayer organic semiconductor interfaces. The diagrams show the energy level alignment for trilayers with physisorbed metal-organic semiconductor (OS) and OS-OS interfaces. Four different cases are shown: a metal-donor-acceptor trilayer for which $\Phi_{\text {met }}<E_{\text {ICT+,D }}<E_{\text {ICT-,A }}$ (panel a), a metal-donor-acceptor trilayer for which $E_{\text {ICT }+ \text {,D }}<E_{\text {ICT-,A }}$ and $E_{\text {ICT }+ \text { D }}<\Phi_{\text {met }}$ (panel $\mathbf{b}$ ), a metal-acceptor-donor trilayer for which $E_{\text {ICT+,D }}<E_{\text {ICT-,A }}<\Phi_{\text {met }}$ and a metal-acceptor-donor trilayer for which $E_{\text {ICT+,D }}<E_{\text {ICT-,A }}$ and $E_{\text {ICT-,A }}>\Phi_{\text {met. }} . \Phi_{\text {met }}$ is the metal work function, and $E_{\text {ICT+,D }}$ and $E_{\text {ICT-,A }}$ correspond to the edges of the donor oxidation and acceptor reduction energy distributions at the interface, respectively. $E_{\mathrm{F}}$, Fermi level; HOMO, highest occupied molecular orbital; LUMO, lowest unoccupied molecular orbital; VL, vacuum level.

Fig. 5 | Dipole potential steps at organic semiconductor-organic semiconductor interfaces. The diagrams show the energy level alignment of two different physisorbed donor-acceptor interfaces. a | When an initially vacuum aligned donor-acceptor interface is modified by an interlayer, it leads to the formation of an intrinsic dipole potential step $\left(\Delta_{\text {intdp }}\right)$. b | Donoracceptor interface for which $E_{\text {ICT+,D }}<E_{\text {ICT-,A }}$, such that electron transfer from the donor to the acceptor occurs at the interface, equilibrating the chemical potential by inducing a dipole potential step $\left(\Delta_{\mathrm{ICT}}\right)$. The induced integer charge transfer (ICT) density of states are depicted in relation to the bulk oxidation and reduction energy distributions (that is, n-polaron and $\mathrm{p}$ polaron density of states, respectively). $E_{\mathrm{F}}$, Fermi level.

Fig. 6 | Organic semiconductor-dielectric interfaces. a | A self-assembled monolayer (SAM) can be introduced between the dielectric and organic semiconductor (OS) layers in an organic field-effect transistor (OFET). The order in the SAM affects the organization of the OS deposited on top of it. $\mathbf{b}$ | The random orientation of the dipole moments in the gate dielectric leads to broadening of the density of states $(N(E)$, where $E$ is energy) in the OS and the localization of polarons; this effect is stronger at the interface than in the bulk. c | Functional groups at the surface of the gate dielectric can trap electrons from the OS. The efficiency of this process increases with increasing polarity of the dielectric surface. d | Plot showing the threshold voltage shift in a polytriarylamine OFET owing to charge trapping. The drain current of the OFET decreases over time The arrows indicate transfer curves measured after certain time periods (between 1 min and 2 weeks). e | Threshold voltage shift induced by the surface dipole potential at the OS-dielectric interface in pentacene OFETs grown on various SAMs. $\mathbf{f} \mid$ Hysteresis induced by the bistable polarization of the ferroelectric dielectric layer within an OFET. HMDS, hexamethyldisilazane; $\mathrm{PS}_{n}$; polystyrene; PS-Ox, $\mathrm{O}_{2}$-plasma treated polystyrene; PVA, polyvinyl alcohol. Panel $\mathbf{b}$ is adapted with permission from ref. ${ }^{134}$, Wiley-VCH, and ref. ${ }^{138}$, ACS. Panel $\mathbf{c}$ is adapted with permission from ref. ${ }^{142}$, ACS. Panel $\mathbf{d}$ is adapted with permission from ref. ${ }^{147}$, Wiley-VCH. Panel $\mathbf{e}$ is adapted from ref. ${ }^{156}$, Springer Nature Limited. Panel $\mathbf{f}$ is adapted with permission from ref. ${ }^{168}$, Springer Nature Limited.

Fig. 8 | Electrochemical processes in organic semiconductors. a | Capacitive (electrical double layer) charge-discharge (top) is characterized by a potential (V)-independent current (I) in cyclic voltammetry, producing a characteristic box-like cyclic voltammogram (middle). The corresponding galvanostatic (constant current) charge-discharge curves show a linear 
dependence of the potential on time (bottom). b | An idealized Faradaic (redox, battery-like) charge-discharge (top) features an anodic peak (corresponding to oxidation, $\mathrm{Red}-\mathrm{e}^{-} \rightarrow \mathrm{Ox}$, where 'Red' and 'Ox' are the reduced and oxidized form of a species, respectively) and a cathodic peak (corresponding to reduction, $\mathrm{Ox}+\mathrm{e}^{-} \rightarrow \mathrm{Red}$ ) around the standard redox potential $E^{0}$ of the redox couple in cyclic voltammetry (middle). Under galvanostatic conditions, chargedischarge occurs at a nearly constant potential $E^{0}$ (bottom). c|An idealized conducting polymer combines both capacitive and Faradaic charge-discharge regimes. However, this is not a simple sum, as the capacitive process necessitates a sufficiently conducting substrate, which is not the case for the undoped state (top). Yellow indicates a poorly conductive state and green a highly conductive one.

Fig. 7 | Organic semiconductor-electrolyte interfaces. The illustrations show the limiting cases of ion movement at the organic semiconductor (OS)-electrolyte interface that accompany redox processes in the OS. Capacitive (electrical double layer) charges and solvent molecules are not shown for clarity. a | When a pristine (undoped) OS is p-doped in the presence of a solution containing mobile anions, the subsequent absorption of solvated counterions results in an increase in mass and swelling. $\mathbf{b}$ | Upon dedoping of a p-doped OS that contains immobile counterions, the absorption of solvated co-ions leads to mass increase and swelling. Yellow indicates a poorly conductive state and green a highly conductive one.

Fig. 9 | Communication between biological organisms and organic electronics. Cells communicate with each other and the outside world predominantly through membrane-bound proteins and receptors. Cells also express adhesion-promoting proteins that enhance adhesion to surfaces and the formation of 3D-structured tissues. Organic electronic surfaces can be chemically modified (panel a) with, for example, $-\mathrm{COOH}$ ligands, to promote the adhesion of biological material. Organic electronic surfaces can also be modified with adhesion-promoting proteins, such as fibronectin, to enhance coupling between cells and the electronic interface. More recent strategies include the formation of composites of organic electronic materials and adhesion-promoting compounds such as lysine or RGD peptides. Dynamic control of adhesion can be achieved through a redox-switching mechanism (panel b), whereby surface-bound proteins are reoriented based on the redox state of the organic (semi)conductor. Beyond control of adhesion, organic electronic surfaces can also control cell and tissue function. A fundamental aspect of this control is the large volumetric capacitance of nano- or microstructured electrodes (panel c), enabling impedance tuning and bulk conductivity dependence on ion concentration. These structures can be tailored to achieve porous, dendritic or even ordered porous (templated) (panel d) films and surfaces. Denser films, such as poly(3-hexylthiophene) , are not porous, leading to well-defined electric double layers (EDLs, panel e) that can be used to precisely control cells or tissue by manipulating local ionic strength. This EDL can be further used by a functionalized (semi)conductor surface with recognition elements, such as DNA probes (panel f), which subtly change the EDL capacitance upon binding of specific nucleic acid targets, enabling precise sensing. Likewise, the gate electrode in transistor devices can be modified with recognition elements (panel g). Finally, many organic (semi)conductors have mixed ionic and electronic conductivity, which enables the transport and delivery of charged species (including metal ions and neurotransmitters (such as acetylcholine, $\mathrm{ACh}^{+}$)) into and through the electrode material (panel h). SAM, self-assembled monolayer. 